\title{
UV Sensitivity in Supersymmetric Large Extra Dimensions: The Ricci-flat Case
}

\author{
C.P. Burgess ${ }^{1,2,3}$ and D. Hoover ${ }^{1}$ \\ 1 Physics Department, McGill University, \\ 3600 University Street, Montréal, Québec, Canada, H3A 2 T8. \\ 2 Department of Physics and Astronomy, McMaster University, \\ 1280 Main Street West, Hamilton, Ontario, Canada, L8S 4 M1. \\ 3 Perimeter Institute, \\ 31 Caroline Street North, Waterloo, Ontario, Canada.
}

\begin{abstract}
We examine the ultraviolet (UV)-sensitive part of the one-loop Casimir energy which is induced when various higher-dimensional supergravities are compactified to $4 \mathrm{D}$ on extra dimensions which are Ricci flat, but otherwise arbitrary. We identify the leading dependence on the mass of very massive higher-dimensional modes, as well as the UV divergent part of the contributions of modes which are massless in the higher-dimensional sense (but which consist of a KK tower of massive modes from the 4D perspective), and show how these are constrained by higher-dimensional general covariance. Some of the implications of co-dimension 2 branes are computed in the limit where their tension is small compared with the extra-dimensional Planck scale (but not small compared with the observed dark energy). Our results support the interpretation of supersymmetric large extra dimensions (SLED) in 6 dimensions as a potential solution to the cosmological constant problem (but do not yet completely clinch the case).
\end{abstract}

This article is dedicated to Bryce DeWitt - a pioneer in the heat-kernel techniques used here - whose recent passing deprives physics of a rare mind.

Keywords: Strings, Branes, Cosmology. 


\section{Contents}

1. Introduction and Conclusions 1

2. General Results 5

2.1 The Gilkey-DeWitt Coefficients 5

2.2 Dimensional Reduction from $6 \mathrm{D}$ to $4 \mathrm{D}$

2.3 Brane-Localized Terms 11

3. Explicit Calculations of Bulk Loops 14

3.1 6D Massless Particles 14

3.2 6D Massive Particles 22

3.3 Supersymmetric Multiplets 23

3.4 Higher-Dimensional Field Content 28

A. Dimensional Regularization and Cutoffs 30

B. The Heat Kernel Coefficients 32

\section{Introduction and Conclusions}

The long-standing problem of understanding why the energy density of the vacuum is unobservably small [1] has recently been recast as the problem of why it is extremely small but has the nonzero value $\rho_{\mathrm{vac}} \approx\left(3 \times 10^{-3} \mathrm{eV}\right)^{4}[2]$. The most challenging part of this problem is to understand why such a small energy density should be 'technically' natural, in the precise sense most clearly enunciated in another context in ref. [3]. It should be emphasized that throughout the history of science all of the many hierarchies of scale which have been encountered - at energies to which we have experimental access - are understood in a technically natural way except the vacuum energy density.

In essence, a small parameter (like the vacuum energy density) is technically natural if its smallness can be understood within the effective theory which describes physics at any scale at which one cares to pose the question. That is, even given that the vacuum energy were small in some microscopic theory of the physics of very short distances, why should it remain small as the physics describing longer distances is 
integrated out? In particular, since integrating out a particle of mass $m$ generates a contribution to $\rho_{\text {vac }}$ which is of order $m^{4},{ }^{1}$ what cancels the huge contributions which are obtained when all of the known particles (whose masses range between $m_{e} \sim 0.5$ $\mathrm{MeV}$ and $m_{t} \sim 180 \mathrm{GeV}$ ) are integrated out? Failure to understand this point seems to indicate that our formulation of physics misses something important at energies above the vacuum-energy scale, $v \sim 10^{-3} \mathrm{eV}$, but in a way which somehow affects only gravitational phenomena.

It may be that progress is being made on this thorny problem, based on the recent recognition that reasonable theories of short distance physics (like string theory) can predict that all of the observed non-gravitational particles are trapped on domain-wall-like surfaces, or branes, which are embedded within spacetime 4 . Such a picture has already dramatically broadened our perspective as to how the lowenergy world could look, such as by introducing the possibility that extra dimensions could be as large as $r \sim 100$ microns across and yet have hitherto escaped detection due to our only being able to search for their existence using gravitational probes. It turns out that such a picture can be viable, although consistency with the observed value of Newton's constant only permits precisely 2 dimensions to be this large [5]. Intriguingly, since $r \sim 100 \mu \mathrm{m}$ implies $\hbar c / r \sim 10^{-3} \mathrm{eV}$, having all presently-observed particles trapped on a 3-brane living within a 6 -dimensional spacetime has the desired effect of dramatically changing gravitational physics at energies above $10^{-3}$ $\mathrm{eV}$, without appreciably changing other non-gravitational phenomena at observable scales.

This observation underlies a recent proposal for understanding the smallness of the vacuum energy density within the framework of 6-dimensional supergravity with the two internal dimensions being sub-millimeter in size: Supersymmetric Large Extra Dimensions (SLED) [6, 7, 8, 9, 10]. In this picture the large extra dimensions contribute in two ways to the explanation of the smallness of the observed $4 \mathrm{D}$ vacuum energy. First, although quantum loops involving the observed particles do give large contributions - $O\left(M^{4}\right)$ for $M \sim \mathrm{TeV}$ - to the $4 \mathrm{D}$ vacuum energy, this energy is localized at the position of the branes on which these particles reside. In many situations the classical gravitational response of the internal dimensions to this localized energy density appears to systematically cancel the brane tensions in the effective $4 \mathrm{D}$ vacuum energy to which cosmology is sensitive. ${ }^{2}$ In this paper we have nothing to say about the on-going investigation of this classical response of the bulk.

\footnotetext{
${ }^{1} \mathrm{~A}$ positive power of the mass rather than the cutoff appears here due to our use throughout of dimensional regularization. The well-known issues of how to understand power-law divergences in dimensional regularization is discussed in Appendix A.

${ }^{2}$ It should be emphasized that the completeness of this cancellation is still under active study. See, for example, ref. [10] for a recent germane analysis.
} 
The second way that the extra dimensions contribute to the effective $4 \mathrm{D}$ vacuum energy density within the SLED proposal is through their quantum response to or Casimir energy due to - the presence of the branes on which ordinary particles live. Given the above-mentioned cancellation of the brane energies with the classical bulk response, it is this quantum response of the bulk which would make up the entirety of the observed Dark Energy density. In particular, it has been argued [6, 8] that within supersymmetric theories this Casimir energy can be naturally as small as $1 / r^{4}$, and so account for the cosmological observations provided $r$ is of order 100 $\mu \mathrm{m} .^{3}$

In this paper we address ourselves to testing whether the quantum response of the extra dimensions can really be this small even though there are large, $O(M)$, bulk states which circulate within loops. In particular, although various one-loop Casimir energies for 6D theories compactified to 4D exist 11 and the results are of order $1 / r^{4}$, these calculations typically only integrate out massless fields in the bulk. However we know that in 4 dimensions it is the integration over the most massive fields which contribute the largest amount to the vacuum energy (recall that contributions to the bulk cosmological constant vary like $m^{n}$ in $n$ dimensions), so we focus here on the quantum effects of bulk fields whose mass satisfies $M \gg 1 / r$. In general the Casimir energy (per unit 4-volume) of such fields can be expected to depend on both $M$ and $r$, and for $M \gg 1 / r$ could generically have the form:

$$
V_{C}(M, r)=c_{0} M^{6} r^{2}+c_{1} M^{4}+\frac{c_{2} M^{2}}{r^{2}}+\frac{c_{3}}{r^{4}}+\ldots
$$

Here the leading contribution depends on $M$ and $r$ as does the contribution of a $6 \mathrm{D}$ cosmological constant. The dimensionless constants, $c_{k}$, are calculable for a given field content in the bulk and on the branes, and the success of the SLED proposal requires the conditions $c_{0}=c_{1}=c_{2}=0$. Our goal in this paper is to track these positive powers of $M$ in the Casimir energy at one loop, to see whether or not they really do vanish.

Fortunately, very general tools exist, based on heat-kernel methods [13, 14, 15, [16], for determining the large- $M$-dependence of the Casimir energy, for a broad choice of fields within the bulk and for a wide variety of geometries for the extra dimensions. We here adapt these techniques to $6 \mathrm{D}$ field theories containing scalars, fermions, gauge bosons, gauge 2-forms, gravitini and gravitons, which are compactified to 4D on an arbitrary Ricci-flat manifold. The restriction to Ricci-flatness is introduced for technical convenience (and is relaxed in a companion paper [12]). Due to the absence of a bulk cosmological constant in 6D supergravity theories this restriction

\footnotetext{
${ }^{3}$ Once factors of $2 \pi$ are included it turns out that $r \sim 10 \mu \mathrm{m}$, and so $\hbar c / r \sim 0.01 \mathrm{eV}$, is required for agreement with the observed Dark Energy density [7].
} 
essentially precludes the study of compactifications in the presence of fluxes, and with spatially-varying scalar fields (which are indeed among the more interesting 6D compactifications [7, 9, 10] for the chiral, gauged supergravities in 6 dimensions). Ricci-flatness remains of interest, however, because it does include a broad class of nontrivial solutions to many $6 \mathrm{D}$ supergravities.

In the heat-kernel treatment all of these dangerous large- $M$ terms arise as local effective interactions within the bulk 6D theory. We use these techniques to identify these effective interactions in a general way for the $6 \mathrm{D}$ fields of most interest. In general bulk loops can also generate large- $M$ effective interactions which are localized at the positions of the branes. We compute the leading dependence of these terms in the limit when the brane tensions are smaller than the 6D gravitational scale (but are much larger than $1 / r)$.

Our final results are as follows. We find that the Casimir energy due to any one 6D field typically does involve terms proportional to $M^{6}, M^{4}$ or $M^{2}$. However our main result is to find that all three of these kinds of terms cancel once they are summed over a massive supermultiplet of $(2,0)$ supersymmetry in 6 dimensions. Furthermore, this cancellation is independent of the details of the compactification from $6 \mathrm{D}$ to $4 \mathrm{D}$ (provided it is Ricci flat), and in particular does not require that the compactification be supersymmetric. This result applies in particular to the contributions of the massive Kaluza-Klein (KK) modes which would be obtained if the 6D supergravity of interest were itself obtained from a 10- (or 11-) dimensional theory by dimensional reduction on 4 (or 5) small dimensions, since these have the same particle content as do the massive $(2,0) 6 \mathrm{D}$ supermultiplets we examine.

As such, we believe these results to be encouraging for the success of the SLED program, inasmuch as they show how bulk supersymmetry can stop very heavy KK particles from contributing too large one-loop amounts to the low-energy effective cosmological constant, both through bulk and brane-localized effective interactions. These results leave one type of one-loop contribution which could still be dangerously large from the SLED point of view: dimension-two interactions localized on the brane (such as a brane-localized Einstein-Hilbert action) which are generated by loops of brane-bound particles (for which supersymmetry is badly broken). Indeed, such effective interactions are likely to be generated by brane loops, and it is not yet clear [10] whether the bulk response need cancel their 4D effects in the same way as it would do for an effective brane tension.

The rest of the paper is organized as follows. Section 2 collects many of the general results on UV sensitivity and Kaluza-Klein theories which prove relevant to the $6 \mathrm{D}$ discussion. Section 3 then uses these results to compute explicitly the UV-sensitive contributions of various 6D massless and massive particles, and assem- 
bles these results into supermultiplets to see how the presence of higher-dimensional supersymmetry changes the generic picture.

\section{General Results}

Our goal is to compute explicitly (as functions of background fields) the ultra-violet sensitive part of the one-loop vacuum energy for compactifications to $4 \mathrm{D}$ of various $6 \mathrm{D}$ field theories. Before embarking on the full calculation it is worth first collecting a few general results concerning the kinds of ultraviolet divergences which can be encountered in calculations of this sort. Because the ultraviolet behavior only depends on the very short-distance limit of the theory these divergences can always be absorbed into renormalizations of local functions of the background fields, with coefficients which can be computed very generally for arbitrary background geometries [13, 14, 15, 16].

\subsection{The Gilkey-DeWitt Coefficients}

This section collects the results for the ultraviolet-divergent parts of the one-loop action obtained by integrating out various kinds of particles in 6 dimensions. To this end, consider a collection of $N$ fields, $\Psi^{z}$ with $z=1, \ldots, N$, coupled to a collection of background fields, possibly including a 6-dimensional spacetime metric, $g_{M N}$, scalars, $\varphi^{i}$, and form fields, $A_{M_{1} . . M_{p}}^{a}$. For each $z, \Psi^{z}$ can carry a gauge and/or Lorentz index, although for simplicity of notation the Lorentz index is suppressed in this section. We suppose that the background-covariant derivative, $D_{M}$, appropriate to $\Psi^{z}$ is:

$$
D_{M} \Psi^{z}=\partial_{M} \Psi^{z}+\omega_{M} \Psi^{z}-i A_{M}^{a}\left(t_{a}\right)_{y}^{z} \Psi^{y}
$$

where $\omega_{M}$ is the appropriate matrix-valued spin connection, and the gauge group is represented by the hermitian matrices $\left(t_{a}\right)^{z}{ }_{y}$. For real fields the $t_{a}$ are imaginary antisymmetric matrices, and (for canonically-normalized gauge bosons) we take the gauge group generators to include a factor of the corresponding gauge coupling, $g_{a}$. The commutator of two such derivatives defines a generalized matrix-valued curvature, $\left(Y_{M N}\right)_{y}^{z} \Psi^{y}=\left[D_{M}, D_{N}\right] \Psi^{z}$, which has the following form: ${ }^{4}$

$$
\left(Y_{M N}\right)_{y}^{z}=\mathcal{R}_{M N} \delta_{y}^{z}-i F_{M N}^{a}\left(t_{a}\right)_{y}^{z}
$$

Here $\mathcal{R}_{M N}$ is the curvature built from the spin connection $\omega_{M}$, which is also related to the Riemann curvature of the background spacetime in a way which is made explicit in what follows.

\footnotetext{
${ }^{4}$ We adopt Weinberg's curvature conventions [17], which differ from those of MTW [18] only in an overall sign in the definition of the Riemann tensor.
} 
Quite generally the result of integrating out the fields $\Psi^{z}$ at one-loop leads to the following contribution to the effective quantum action

$$
i \Sigma=-(-)^{F} \frac{1}{2} \operatorname{Tr} \log \Delta
$$

where $(-)^{F}=1$ for bosons and -1 for fermions, and the differential operator $\Delta^{z}$ has the following form

$$
\Delta_{y}^{z}=-\delta_{y}^{z} \square+X_{y}^{z}+\left(m^{2}\right)_{y}^{z}
$$

Here $\delta^{z}{ }_{y}$ is the Kronecker delta, $\square=g^{M N} D_{M} D_{N}$ and $X_{y}^{z}$ is a local quantity built from the background fields whose form depends on the kind of field under consideration (explicit examples are given below for the usual fields of interest). The mass matrix, $m^{2}$, can either be regarded as being physical masses which are extracted from within $X$, or as a regulator mass, $\left(m^{2}\right)_{y}^{z}=\mu^{2} \delta_{y}^{z}$, which is to be taken to zero (or to infinity) at the end of the calculation.

Our interest for this section is in two parts of $\Sigma$ which are very closely related to one another. One of these is the ultraviolet divergent part of $\Sigma$, and the other is that part of $\Sigma$ which depends most strongly on the mass of any massive 6D fields which are integrated out. We collect here the very general results which can be obtained for both of these quantities using the Gilkey-DeWitt heat-kernel methods [13, 14, 15, 16]. When identifying the divergent part we work within dimensional regularization and so continue the spacetime dimension to complex values, $n$, which are slightly displaced from the actual integer spacetime dimension, 6, which is of interest: $n=6-2 \epsilon$. We then follow the poles in $\Sigma$ as $\epsilon \rightarrow 0$, in the usual fashion. These may be related to the logarithmic divergences which would be obtained from an ultraviolet cutoff, $\Lambda$, through the usual relation (see Appendix A)

$$
\frac{1}{\epsilon} \leftrightarrow \ln \left(\Lambda^{2}\right)
$$

For 6D spaces without boundaries and singularities the ultraviolet-divergent terms (and heavy-mass-dependent terms) are simply characterized. In $n$ dimensions they may be written as [13, 16]

$$
\Sigma_{\infty}=\frac{1}{2}(-)^{F}\left(\frac{1}{4 \pi}\right)^{n / 2} \int d^{n} x \sqrt{-g} \sum_{k=0}^{[n / 2]} \Gamma(k-n / 2) \operatorname{Tr}\left[m^{n-2 k} a_{k}\right]
$$

which for $n=6-2 \epsilon$ specializes to

$$
\Sigma_{\infty}=\frac{1}{2(4 \pi)^{3}}(-)^{F} \sum_{k=0}^{3} \Gamma(k-3+\epsilon) \int d^{6} x \sqrt{-g} \operatorname{Tr}\left[m^{6-2 k} a_{k}\right]
$$


Here $\Gamma(z)$ denotes Euler's gamma function. The divergence as $\epsilon \rightarrow 0$ is contained within the gamma function, which has poles at non-positive integers of the form $\Gamma(-r+\epsilon)=(-)^{r} /(r ! \epsilon)+\cdots$, for $\epsilon$ an infinitesimal and $r$ a non-negative integer. The coefficients, $a_{k}$, are known $N^{\prime} \times N^{\prime}$ matrix-valued local quantities constructed from the background fields, to which we return below. Here $N^{\prime}=N d$ with $N$ counting the number of fields and $d$ being the dimension of the appropriate Lorentz representation. The trace is over the $N^{\prime}$ matrix indices of the $a_{k}$.

The above expression shows that for massless fields $(m=0)$ in compact spaces without boundaries and singularities in 6 dimensions the divergent contribution is proportional to $\operatorname{tr}\left[a_{3}\right]$ in dimensional regularization, so the problem of identifying these divergences reduces to the construction of this coefficient. ${ }^{5}$ By contrast, for massive fields there are divergences proportional to $\operatorname{tr}\left[m^{6} a_{0}\right], \operatorname{tr}\left[m^{4} a_{1}\right], \operatorname{tr}\left[m^{2} a_{2}\right]$ and $\operatorname{tr}\left[a_{3}\right]$, and these are also the terms in $\Sigma$ which involve the highest powers of $m$. For example, it turns out that $a_{0}$ is proportional to the unit matrix, $I$, and so the term involving $a_{0}$ represents a divergent and strongly $m$-dependent contribution to the $6 \mathrm{D}$ cosmological constant, proportional to $\operatorname{tr}\left[\mathrm{m}^{6}\right]$. Similarly, since $a_{1}$ contains a term proportional to $R I$, where $R$ is the background metric's Ricci scalar, $\operatorname{tr}\left[m^{4} a_{1}\right]$ contains an $m$-dependent renormalization of the Einstein-Hilbert action, and so on.

What is attractive about the above results is that an algorithm for constructing the coefficients $a_{k}$ is known for general $X$ and $D_{M}$, and the result for the first few has been computed explicitly [13, 16] and can be given as a closed form in terms of $X$, background curvatures and the generalized curvature $\left(Y_{M N}\right)^{z}{ }_{y}$. The explicit results for the quantities $a_{0}$ through $a_{3}$ are given in their general form in Appendix B. These allow the calculation of the most ultraviolet-sensitive contributions from quantum loops for arbitrary theories in the presence of very general background field configurations.

The remainder of this section specializes these results to the various fields of interest for $6 \mathrm{D}$ supergravity theories. We take the bosonic part of the action for these theories to be

$$
\begin{aligned}
S=-\int d^{6} x \sqrt{-g}\left[\frac{1}{2} g^{M N} G_{i j}(\Phi) D_{M} \Phi^{i} D_{N} \Phi^{j}+V(\Phi)\right. & \\
& \left.+\frac{1}{2} U(\Phi) R+\sum_{p} \frac{1}{2 p !} W_{p}(\Phi) F_{M_{1} . . M_{p}}^{a} F_{a}^{M_{1} . . M_{p}}\right],
\end{aligned}
$$

where $\Phi^{i}$ denote the theory's scalar fields, and $F_{(p)}=\mathrm{d} A_{(p-1)}+\omega_{p}$ is a $p$-form field strength for a $(p-1)$-form gauge potential, and $\omega_{p}$ is an appropriate Chern-Simons

\footnotetext{
${ }^{5}$ Notice that the freedom to keep $m^{2}$ within or separate from $X$ implies that the divergence obtained from computing just $a_{3}$ using $X_{m}=X+m^{2}$ gives the same result as computing $a_{0}$ through $a_{3}$ using only $X$.
} 
form whose details are not important in what follows. The coefficient functions $U$, $V, W_{p}$ and $G_{i j}$ are known functions of the $\Phi^{i}$ which differ for different choices for the $6 \mathrm{D}$ supergravity of interest.

As usual, we are always free to use the classical equations of motion obtained from this action to simplify the one-loop quantity $\Sigma$, because anything which vanishes with the classical field equations may be removed from $\Sigma$ by performing an appropriate field redefinition [28, 29]. For simplicity we also specialize here to the case where these classical equations are the same as those for pure gravity, $R_{M N}=0$, and so for which all other fields have trivial stress energy: $F_{M_{1} . . M_{p}}=\partial_{M} \Phi^{i}=0$. We return to the more general case with more complicated classical solutions in a companion paper [12].

\subsection{Dimensional Reduction from 6D to 4D}

When using the Gilkey coefficients in 6-dimensional theories compactified to 4 dimensions one might be tempted to ask whether we should take $n=4$ or $n=6$ when evaluating formulae like eq. (2.6). In this section we show that it makes no difference, inasmuch as the sum over the result for each 4D KK mode reproduces the full $6 \mathrm{D}$ expression.

\section{Dimensional Reduction on $S^{2}$}

To establish this point we take the simplest nontrivial example: the reduction of a $6 \mathrm{D}$ scalar field theory to $4 \mathrm{D}$ on a 2 -sphere. ${ }^{6}$ For these purposes we start with the $6 \mathrm{D}$ action

$$
S=-\int d^{4} x d^{2} y \sqrt{-g}\left(-\Phi^{*} \square_{6} \Phi+M^{2} \Phi^{*} \Phi\right)
$$

where $\Phi$ is a minimally-coupled complex scalar field with a $6 \mathrm{D}$ mass $M$, and $\square_{6}=$ $g^{M N} D_{M} D_{N}$ is the $6 \mathrm{D}$ d'Alembertian.

We further assume that the background metric takes the product form $\mathrm{d} s_{6}^{2}=$ $g_{\mu \nu}(x) \mathrm{d} x^{\mu} \mathrm{d} x^{\nu}+g_{m n}(y) \mathrm{d} y^{m} \mathrm{~d} y^{n}$, where $g_{m n}$ is the internal two dimensions and $g_{\mu \nu}$ is the metric of the 'large' 4 dimensions. In this case the $6 \mathrm{D}$ d'Alembertian is related to its $4 \mathrm{D}$ counterpart, $\square_{4}=g^{\mu \nu} D_{\mu} D_{\nu}$, and the $2 \mathrm{D}$ Laplacian, $\square_{2}=g^{m n} D_{m} D_{n}$, by $\square_{6}=\square_{4}+\square_{2}$. Finally, we specialize to an internal $S^{2}$ by taking $g_{m n} \mathrm{~d} y^{m} \mathrm{~d} y^{n}=$ $r^{2} \gamma_{m n} \mathrm{~d} y^{m} \mathrm{~d} y^{n}=r^{2}\left(\mathrm{~d} \theta^{2}+\sin ^{2} \theta \mathrm{d} \varphi^{2}\right)$, where $r$ denotes the 2-sphere's radius.

The dimensional reduction is performed by writing $\Phi$ as a mode sum in terms of the eigenfunctions of the scalar Laplacian on a 2-sphere. Our ansatz therefore

\footnotetext{
${ }^{6}$ Compactification on a torus is too trivial for the present purposes, since all of the Gilkey coefficients except $a_{0}$ tend to vanish for flat manifolds like torii. Ref. [11] includes more recent explicit examples.
} 
becomes:

$$
\Phi(x, y)=\frac{1}{r} \sum_{l=0}^{\infty} \sum_{m=-l}^{l} \phi_{l}^{m}(x) Y_{l}^{m}(y),
$$

where $Y_{l}^{m}(y)$ are the standard spherical harmonics, and $\phi_{l}^{m}(x)$ are the corresponding 4D fields. Using $\square_{6}=\square_{4}+\square_{2}$ we find

$$
\Phi^{*} \square_{6} \Phi=\frac{1}{r^{2}} \sum_{m, l, m^{\prime}, l^{\prime}}\left[\left(\phi_{l}^{m *}(x) \square_{4} \phi_{l^{\prime}}^{m^{\prime}}(x)\right) Y_{l}^{m *} Y_{l^{\prime}}^{m^{\prime}}+\phi_{l}^{m *}(x) \phi_{l^{\prime}}^{m^{\prime}}(x) Y_{l}^{m *} \square_{2} Y_{l^{\prime}}^{m^{\prime}}\right] .
$$

Finally, using the results $-\square_{2} Y_{l}^{m}=\left[l(l+1) / r^{2}\right] Y_{l}^{m}, \sqrt{-g_{6}}=r^{2} \sqrt{-g_{4}} \sqrt{\gamma}$ as well as the orthonormality relations $\int d^{2} y \sqrt{\gamma} Y_{l}^{m *} Y_{l^{\prime}}^{m^{\prime}}=\delta_{l l^{\prime}} \delta_{m m^{\prime}}$, the $6 \mathrm{D}$ action becomes

$$
S=-\sum_{m, l} \int d^{4} x \sqrt{-g_{4}}\left[-\phi_{l}^{m *} \square \phi_{l}^{m}+\left(M^{2}+\frac{l(l+1)}{r^{2}}\right) \phi_{l}^{m *} \phi_{l}^{m}\right],
$$

where all quantities are now functions only of $x$. This is the standard manipulation which expresses the theory of one complex $6 \mathrm{D}$ scalar in terms of an infinite tower of 4D Kaluza-Klein (KK) modes, consisting of complex 4D scalars whose 4D masses are $\mu_{l}^{2}=M^{2}+l(l+1) / r^{2}$. Notice that for scalars on the 2 -sphere each KK mass level has degeneracy $d_{l}=(2 l+1)$.

\section{UV Sensitivy}

We now check that the UV sensitive terms are identical when computed in 6 dimensions or as the sum over a series of $4 \mathrm{D}$ results for each KK mode. Recall for these purposes that the divergent part of the one-loop quantum action can be written in $n$ dimensions as

$$
\Sigma_{\infty}=\frac{1}{2}(-)^{F}\left(\frac{1}{4 \pi}\right)^{n / 2} \int d^{n} x \sqrt{-g} \sum_{k=0}^{[n / 2]} \Gamma(k-n / 2) \operatorname{Tr}\left[M^{n-2 k} a_{k}\right],
$$

where $M$ is the $n$-dimensional mass of the particle which traverses the loop.

The $6 D$ Calculation: For the $6 \mathrm{D}$ calculation we use the general result specialized to a minimally-coupled scalar field in $n=6$ dimensions. For simplicity we also assume the $6 \mathrm{D}$ complex scalar to be massless - so $M=0-$ and take $Y_{M N}=X=0$. Because the scalar has been taken to be massless in 6D, the only relevant Gilkey coefficient is $a_{3}$, which we must evaluate. For this evaluation we specialize the general result to the product geometry, for which $R_{6}=R_{4}+R_{2}, R_{M N} R^{M N}=R_{\mu \nu} R^{\mu \nu}+R_{m n} R^{m n}$ etc., where the 2 -sphere curvatures satisfy $R_{m n p q}=\left(1 / r^{2}\right)\left(g_{m q} g_{n p}-g_{m p} g_{n q}\right), R_{m n}=$ $-\left(1 / r^{2}\right) g_{m n}$ and $R_{2}=-2 / r^{2}$, so $R_{m n p q} R^{m n p q}=2 R_{m n} R^{m n}=R_{2}^{2}=4 / r^{4}$. 
Remembering the overall factor of 2 because the scalar is complex, and expanding $a_{3}$ in powers of the $4 \mathrm{D}$ curvature tensor we find

$$
\operatorname{Tr}\left[a_{3}\right]=\frac{8}{315 r^{6}}-\frac{1}{45 r^{4}} R_{4}+\ldots
$$

and so integrating over the 2 -sphere, and using $\Gamma(0) \sim 1 / \epsilon$, we find

$$
\Sigma_{\infty}=\frac{1}{2}\left(\frac{1}{4 \pi}\right)^{2} \frac{1}{\epsilon} \int d^{4} x \sqrt{-g}\left(\frac{8}{315 r^{4}}-\frac{1}{45 r^{2}} R_{4}+\ldots\right) .
$$

The $4 D$ Calculation: In the $4 \mathrm{D}$ theory we may similarly take $X=0$ provided we separate explicitly the KK mass terms from $X$, and up to linear order in $R_{4}$ we need keep only the contributions to $a_{1}$ and $a_{0}$. Using $\Gamma(-r+\epsilon) \sim(-)^{r} /(r ! \epsilon)$ we find for each KK mode (remembering again the factor of 2 for complex scalars),

$$
\begin{aligned}
\Sigma_{\infty}^{l m} & =\frac{1}{2}\left(\frac{1}{4 \pi}\right)^{2} \int d^{4} x \sqrt{-g}\left(\operatorname{Tr}\left[\mu_{l}^{4} a_{0}\right] \frac{1}{2 \epsilon}-\operatorname{Tr}\left[\mu_{l}^{2} a_{1}\right] \frac{1}{\epsilon}+\ldots\right) \\
& =\frac{1}{2}\left(\frac{1}{4 \pi}\right)^{2} \int d^{4} x \sqrt{-g}\left(\frac{\mu_{l}^{4}}{\epsilon}+\frac{\mu_{l}^{2}}{3 \epsilon} R_{4}+\ldots\right) .
\end{aligned}
$$

We now sum over the KK modes, and interpret the resulting divergent sums using $\zeta$-function regularization [19, 20, 21]. Recalling that each mass eigenvalue $\mu_{l}^{2}=l(l+1) / r^{2}$ has degeneracy $(2 l+1)$ we have

$$
\begin{aligned}
\sum_{l m} \mu_{l}^{4} & =\sum_{l=0}^{\infty}\left(\frac{l(l+1)}{r^{2}}\right)^{2}(2 l+1) \\
& =\frac{1}{r^{4}} \sum_{l=1}^{\infty}\left(2 l^{5}+5 l^{4}+4 l^{3}+l^{2}\right) \\
& =\frac{1}{r^{4}}[2 \zeta(-5)+5 \zeta(-4)+4 \zeta(-3)+\zeta(-2)]
\end{aligned}
$$

where $\zeta(s)=\sum_{n=1}^{\infty}\left(1 / n^{s}\right)$ is the Riemann zeta-function. Using the following results 22

$$
\begin{aligned}
& \zeta(-5)=-\frac{1}{252}, \zeta(-3)=\frac{1}{120} \\
& \zeta(-4)=\zeta(-2)=0, \zeta(-1)=-\frac{1}{12}
\end{aligned}
$$

we find that

$$
\sum_{l m} \mu_{l}^{4}=\frac{8}{315 r^{4}}
$$


Similarly

$$
\begin{aligned}
\sum_{l m} \mu_{l}^{2} & =\sum_{l=0}^{\infty} \frac{l(l+1)}{r^{2}}(2 l+1) \\
& =\frac{1}{r^{2}} \sum_{l=1}^{\infty}\left(2 l^{3}+3 l^{2}+l\right) \\
& =\frac{1}{r^{2}}[2 \zeta(-3)+3 \zeta(-2)+\zeta(-1)], \\
& =-\frac{1}{15 r^{2}} .
\end{aligned}
$$

Finally, combining these results we obtain the following expression for the divergent piece, as computed in 4 dimensions:

$$
\begin{aligned}
\Sigma_{\infty} & =\sum_{l m} \Sigma_{\infty}^{l m} \\
& =\frac{1}{2}\left(\frac{1}{4 \pi}\right)^{2} \frac{1}{\epsilon} \int d^{4} x \sqrt{-g}\left(\frac{8}{315 r^{4}}-\frac{1}{45} R_{4}+\ldots\right) .
\end{aligned}
$$

As expected, we obtain the same result for $\Sigma_{\infty}$ regardless of whether we do the calculation in the $6 \mathrm{D}$ or the $4 \mathrm{D}$ theory, provided we sum over all of the KK modes in the lower-dimensional case. It is therefore a matter only of convenience whether or not to use the higher- or lower-dimensional formulation.

\section{Dimensional Reduction in Supersymmetric Models}

The previous calculations are useful when computing the UV sensitivity of 6D supersymmetric theories, particularly when $6 \mathrm{D}$ supersymmetry breaks due to the compactification down to 4 dimensions. Seen from the $4 \mathrm{D}$ point of view it might appear that supersymmetry is badly broken, making the cancellations due to 6D supersymmetry hard to follow. However, the freedom to perform computations in the higher dimensions makes it easier to see the cancellations which follow from higher-dimensional supersymmetry. Physically these cancellations still hold because it is the UV sensitive part of the one-loop result which we compute, and this is only sensitive to the very short wavelengths for which the higher-dimensional symmetries apply.

We now turn to a discussion those UV-sensitive effects which are localized near the position of any co-dimension 2 branes.

\subsection{Brane-Localized Terms}

For supersymmetric large extra dimensions we require the Casimir energy in the presence of brane sources, which typically introduces either boundaries or singularities into the bulk geometry, depending on the dimension of the brane involved. Since 
the presence of boundaries and singularities permit the appearance of more complicated divergences in the Casimir energy, additional local counter-terms are required in order to renormalize them. Since all of these are localized at the brane positions, they can be regarded as renormalizations of the effective brane actions.

Unfortunately, results with the generality described above are not yet available in the presence of co-dimension 2 brane sources. Some things are known, however, and we summarize those which are most relevant to the SLED proposal here. The main calculations which have been done assume the geometry near the branes to be described by a conical singularity, for which the $2 \mathrm{D}$ bulk metric can be written in the form $\mathrm{d} r^{2}+c_{b}^{2} r^{2} \mathrm{~d} \theta^{2}$ near the singularity $(r=0)$, where $\theta$ is a periodic coordinate with period $2 \pi$ and $c_{b}$ is a constant. This geometry has a defect angle at the brane position, whose size is given by $\delta=2 \pi\left(1-c_{b}\right)$. This introduces a delta-function-type divergence into the curvature at the brane position which is proportional to $\delta$. This kind of singularity is often (but not always [23, 24]) what is produced by 3-branes which are aligned within the 6 dimensions to be parallel with the large 4 dimensions.

Some explicit results are known for the types of ultraviolet divergences which arise in this case. This includes explicit results for the heat kernel coefficients for specific types of particles in the presence of these singularities [16, 25] as well as more general expressions which apply to the limit of small defect angles, which are obtained by interpreting the cone to be the limit of a sequence of 'blunted' cones for each of which the tip is smoothed off [26, 27]. According to this line of argument, the leading contributions (for small defect angles) to the brane counter-terms may be found by taking the limit of the bulk terms obtained for each of the blunted cones.

Applied to quadratic order in the background curvatures, this leads to the relations:

$$
\begin{aligned}
& \int_{\tilde{B}} d^{6} x \sqrt{-g} \tilde{R} \approx \int_{B^{\prime}} d^{6} x \sqrt{-g} R-\sum_{b} 4 \pi\left(1-c_{b}\right) \int_{b} d^{4} x \sqrt{-h} \\
& \int_{\tilde{B}} d^{6} x \sqrt{-g} \tilde{R}^{2} \approx \int_{B^{\prime}} d^{6} x \sqrt{-g} R^{2}-\sum_{b} 8 \pi\left(1-c_{b}\right) \int_{b} d^{4} x \sqrt{-h} R \\
& \int_{\tilde{B}} d^{6} x \sqrt{-g} \tilde{R}_{M N} \tilde{R}^{M N} \approx \int_{B^{\prime}} d^{6} x \sqrt{-g} R_{M N} R^{M N} \\
& \int_{\tilde{B}} d^{6} x \sqrt{-g} \tilde{R}_{M N L P} \tilde{R}^{M N L P} \approx \int_{B^{\prime}} d^{6} x \sqrt{-g} R_{M N L P} R^{M N L P} \\
&-\sum_{b} 8 \pi\left(1-c_{b}\right) \int_{b} d^{4} x \sqrt{-h} R_{a a}
\end{aligned}
$$

where the approximate equality indicates that terms of order $\left(1-c_{b}\right)^{2}$ are neglected 
on the right-hand side. In these expressions $\tilde{B}$ denotes the limit of the sequence of blunted cones (having curvature $\tilde{R}_{M N L P}$ ) which approach the bulk, $B$, including the conical singularity, and $b$ denotes the $4 \mathrm{D}$ world-surface of the brane, defined by the position of the conical singularity. $B^{\prime}$ denotes the bulk with the positions of the conical singularities removed: $B^{\prime}=B-\sum_{b} b$, and $R_{M N L P}$ is the curvature of this bulk in the limit of no singularity. $h_{\mu \nu}$ denotes the induced metric on $b$, which we also suppose to have no extrinsic curvature, and

$$
R_{a a}=\sum_{a=1}^{2} R_{M N} n_{a}^{M} n_{a}^{N}, \quad \text { and } \quad R_{a b a b}=\sum_{a, b=1}^{2} R_{M N P Q} n_{a}^{M} n_{b}^{M} n_{a}^{P} n_{b}^{Q}
$$

where $n_{i}^{M}$ denote two mutually-orthogonal unit normals to the appropriate brane world surface.

Because these expressions for the brane-localized contributions to the heat kernel are obtained as limits of a sequence of bulk contributions, they permit an easy generalization of the expressions given in Appendix B to include brane-localized terms in the limit of small defect angles. We now summarize the results which are obtained in this way, which give the following brane-localized counterterms:

$$
\begin{gathered}
\int_{\tilde{B}} d^{6} x \sqrt{-g} \operatorname{Tr}\left[m^{4} a_{1}\right] \approx \int_{B^{\prime}} d^{6} x \sqrt{-g} \operatorname{Tr}\left[m^{4} a_{1}\right]+\sum_{b} \frac{2 \pi}{3}\left(1-c_{b}\right) \int_{b} d^{4} x \sqrt{-h} \operatorname{Tr}\left[m^{4}\right] \\
\int_{\tilde{B}} d^{6} x \sqrt{-g} \operatorname{Tr}\left[m^{2} a_{2}\right] \approx \int_{B^{\prime}} d^{6} x \sqrt{-g} \operatorname{Tr}\left[m^{2} a_{2}\right] \\
-\sum_{b} \frac{2 \pi}{3}\left(1-c_{b}\right) \int_{b} d^{4} x \sqrt{-h}\left\{\operatorname{Tr}\left[m^{2} \hat{X}\right]\right. \\
\left.+\frac{1}{30}\left(2 R_{a b a b}-R_{a a}+5 R\right) \operatorname{Tr}\left[m^{2}\right]\right\} .
\end{gathered}
$$

Here $\operatorname{Tr}\left[m^{2} \hat{X}\right]$ is defined as follows: if $\operatorname{Tr}\left[m^{2} X\right]=a R+b$ then $\operatorname{Tr}\left[m^{2} \hat{X}\right]=2 a R+b$.

These expressions assume that the defect angles are small and that the only singular bulk fields near the brane positions are the curvatures. This latter assumption is a natural consequence of our assumption of the vanishing of background quantities like $X$ and $F_{M N}^{a} F_{a}^{M N}$, since this guarantees that these quantities remain smooth there. They predict (for small defect angles) the new brane-localized ultraviolet divergences which arise once branes are inserted into the bulk space.

Where the explicit results can be compared with this small-defect limit they agree. $^{7}$

${ }^{7}$ It is claimed in ref. 27] that the small-defect result does not agree with explicit calculations for the divergences produced by integrating out spin-3/2 and spin- 2 particles. However we regard these conclusions to be suspect inasmuch as the obstruction they find explicitly involves the contributions of pure-gauge modes - i.e. conformal Killing vectors and spinors - and this reference does not treat properly the contributions of the ghosts which would be expected to cancel such modes. 
Finally, since our present interest is in whether the Gilkey coefficients vanish when summed over the elements of a $6 \mathrm{D}$ supermultiplet, it is useful to notice here that - to linear order in $\left(1-c_{b}\right)$ - the vanishing of the UV sensitive terms on the boundary is an automatic consequence of the vanishing of all of the corresponding bulk terms from which they arise. It would clearly be very useful to have more general calculations of these quantities.

\section{Explicit Calculations of Bulk Loops}

This section specializes these general results for the ultraviolet-divergent parts of the one-loop action to several specific particle types which arise in 6 dimensional supergravities. For technical reasons we specialize in what follows to the case where all background gauge fields vanish and - with applications to higher-dimensional supergravity in mind - where the higher-dimensional cosmological constant vanishes. In this situation the classical field equations imply the background metric must satisfy $R_{M N}=0$, and so we also restrict to Ricci-flat metrics.

\subsection{D Massless Particles}

We start by computing the results for $6 \mathrm{D}$ massless particles. Although we specialize in the end to vanishing gauge fields and to Ricci-flat metrics, for later convenience we quote our intermediate results for lower-spin fields in the more general case where the gauge fields are nonzero and the metric is arbitrary.

\section{Scalars}

The general scalar-field action given above involves a collection of $N$ real scalar fields, $\Phi^{i}$, coupled to a background spacetime metric, $g_{M N}$, and form-fields, $A_{M_{1} . . M_{p-1}}^{a}$. Assuming that the scalars couple to a background (1-form) gauge potential, the background covariant derivative appropriate to this case is:

$$
D_{M} \Phi^{i}=\partial_{M} \Phi^{i}-i A_{M}^{a}\left(t_{a}\right)_{j}^{i} \Phi^{j}
$$

where the matrices $\left(t_{a}\right)_{j}^{i}, i=1 \ldots N$, represent the gauge group on the scalars.

To compute the one-loop quantum effects of scalar fluctuations we linearize this action about a particular background configuration, $\varphi^{i}$, according to: $\Phi^{i}=\varphi^{i}+\phi^{i}$, where $\partial_{M} \varphi=0$. Expanding the classical action to quadratic order in $\phi^{i}$ allows the identification of the operator $\Delta^{i}$, which is given by

$$
\Delta^{i}{ }_{j}=-\delta^{i}{ }_{j} \square+X^{i}{ }_{j},
$$


with $X^{i}{ }_{j}$ given by

$$
X_{j}^{i}=G^{i k}\left[V_{k j}(\varphi)+\frac{1}{2} R U_{k j}+\frac{1}{4} F_{M N}^{a} F_{a}^{M N} W_{k j}(\varphi)\right]
$$

and

$$
\left(Y_{M N}\right)^{i}{ }_{j}=-i F^{a}{ }_{M N}\left(t_{a}\right)_{j}^{i}
$$

In this last expression the subscripts on $U, V$ and $W$ denote differentiation with respect to the background field $\varphi^{i}$.

Specializing to Ricci-flat geometries with vanishing Maxwell fields, these simplify to $X^{i}{ }_{j}=G^{i k} V_{k j}$ and $Y_{m n}=0$. We will also make use of the following result for a Ricci flat space (we neglect total derivatives)

$$
D_{E} R_{A B C D} D^{E} R^{A B C D}=-I_{1}-4 I_{2}
$$

where $I_{1}$ and $I_{2}$ are defined as

$$
\begin{aligned}
& I_{1}=R_{A B C D} R_{E F}^{A B} R^{C D E F} \\
& I_{2}=R_{A B C D} R_{E F}^{A C} R^{B E D F} .
\end{aligned}
$$

It should be noted that the integrand of the Euler number, $\chi$, in 6 dimensions is proportional to the combination $\left(-I_{1}+2 I_{2}\right)$.

With these choices, the contributions to the $a_{k}$ due to a loop of scalars are given by $N \times N$ matrices, with $a_{0}$ through $a_{3}$ given by

$$
\begin{aligned}
& a_{0}=I \\
& a_{1}=-X \\
& a_{2}=\frac{1}{180} R_{A B M N} R^{A B M N}+\frac{1}{2} X^{2} \\
& a_{3}=-\frac{17}{45360} I_{1}+\frac{1}{1620} I_{2}+\frac{1}{360}\left(-60 X^{3}-2 X R_{A B M N} R^{A B M N}+30 X \square X\right)
\end{aligned}
$$

For instance, for the particular case $V_{j k}=0$ we have $X=0$ and

$$
\begin{aligned}
& (-)^{F} \operatorname{tr}_{0}\left[a_{0}\right]=N, \quad(-)^{F} \operatorname{tr}_{0}\left[a_{1}\right]=0 \\
& (-)^{F} \operatorname{tr}_{0}\left[a_{2}\right]=\frac{N}{180} R_{A B M N} R^{A B M N} \\
& (-)^{F} \operatorname{tr}_{0}\left[a_{3}\right]=-\frac{17 N}{45360} I_{1}+\frac{N}{1620} I_{2}
\end{aligned}
$$




\section{Spinors}

For $N$ symplectic-Weyl spinors in 6 dimensions, $\psi^{a}$ with $a=1, \ldots, N$, the action can be taken to be,

$$
S=-\frac{1}{2} \int d^{6} x \sqrt{-g} G_{a b}(\varphi) \bar{\psi}^{a} \not D \psi^{b},
$$

where $\not D=e_{A}{ }^{M} \Gamma^{A} D_{M}$ with $\Gamma^{A}$ being the $6 \mathrm{D}$ Dirac matrices and $e_{A}{ }^{M}$ denoting the inverse of the sechsbein, $e_{M}{ }^{A}$, which satisfies $e_{M}{ }^{A} e_{N}{ }^{B} \eta_{A B}=g_{M N}$. Since $6 \mathrm{D}$ symplectic-Weyl spinors have 2 complex components their representation of the $6 \mathrm{D}$ Lorentz group has $d=4$ real dimensions.

The differential operator which governs the one-loop contributions is in this case $\not D=e_{A}{ }^{M} \Gamma^{A} D_{M}$ and so in order to use the general results of the previous section we write (assuming there are no gauge or Lorentz anomalies)

$$
\log \operatorname{det} \not D=\frac{1}{2} \log \operatorname{det}\left(-\not D^{2}\right)
$$

which implies

$$
\begin{aligned}
i \Sigma_{1 / 2} & =\frac{1}{2} \operatorname{Tr} \log \not D=\frac{1}{4} \operatorname{Tr} \log \left(-\not D^{2}\right) \\
& =\frac{1}{4} \operatorname{Tr} \log \left(-\square-\frac{1}{4} R+\frac{i}{2} \Gamma^{A B} F_{A B}^{a} t_{a}\right),
\end{aligned}
$$

where $\Gamma_{A B}=\frac{1}{2}\left[\Gamma_{A}, \Gamma_{B}\right]$ and $t_{a}$ denotes the gauge-group generator acting on the spinor fields. This allows us to adopt the previous results for the ultraviolet divergences, provided we divide the result by an overall factor of 2 , and use

$$
X=-\frac{1}{4} R I+\frac{i}{2} \Gamma^{A B} F_{A B}^{a} t_{a}
$$

where $I$ is the $\mathcal{N} \times \mathcal{N}$ unit matrix, with $\mathcal{N}=N d$. Similarly, we find

$$
Y_{M N}=-\frac{1}{4} R_{M N A B} \Gamma^{A B}-i F^{a}{ }_{M N} t_{a}
$$

and $\mathrm{so}^{8}$

$$
\operatorname{Tr}_{1 / 2}\left[Y_{M N} Y^{M N}\right]=-4 \operatorname{tr}_{1 / 2}\left(t_{a} t_{b}\right) F_{M N}^{a} F^{b M N}-\frac{N}{2} R_{A B M N} R^{A B M N} .
$$

Keeping explicit the sign due to statistics, and dropping terms which vanish when traced, this leads to the following expressions for the divergent contributions

\footnotetext{
${ }^{8}$ We adopt the convention of using $\operatorname{Tr}[. .$.$] to denote a trace which includes the Lorentz and/or$ spacetime indices, while reserving $\operatorname{tr}[\ldots]$ for those which run only over the 'flavor' indices which count the fields of a given spin.
} 
of $N$ 6D Weyl fermions, where we now specialize to a Ricci flat background with vanishing gauge fields:

$$
\begin{aligned}
& (-)^{F} \operatorname{Tr}_{1 / 2}\left[a_{0}\right]=-2 N, \quad(-)^{F} \operatorname{Tr}_{1 / 2}\left[a_{1}\right]=0, \\
& (-)^{F} \operatorname{Tr}_{1 / 2}\left[a_{2}\right]=\frac{7 N}{720} R_{A B M N} R^{A B M N} \\
& (-)^{F} \operatorname{Tr}_{1 / 2}\left[a_{3}\right]=-\frac{29 N}{45360} I_{1}+\frac{N}{648} I_{2} .
\end{aligned}
$$

\section{Gauge Bosons}

For $N$ gauge bosons, $\mathcal{A}_{M}^{a}$, with field strength $\mathcal{F}_{M N}^{a}$ and $a=1, \ldots, N$, the action is

$$
S=-\int d^{6} x \sqrt{-g} \frac{1}{4} W_{2}(\varphi) \mathcal{F}_{M N}^{a} \mathcal{F}_{a}^{M N}
$$

expanded to quadratic order about the background fields: $\mathcal{A}_{M}^{a}=A_{M}^{a}+\delta A_{M}^{a}$.

Adding a gauge-averaging term proportional to $\xi^{-1} W_{2}(\varphi)\left(D^{M} \mathcal{A}_{M}^{a}\right)^{2}$, it is possible to choose the gauge parameter $\xi$ such that the one-loop contribution due to the gauge fields has the standard form, with the differential operator governing the loop given by

$$
\Delta^{a M}{ }_{b N}=-\delta^{a}{ }_{b} \delta^{M}{ }_{N} \square+X^{a M}{ }_{b N},
$$

with

$$
X^{a M}{ }_{b N}=-R^{M}{ }_{N} \delta_{b}^{a}+2 i\left(\tau_{c}\right)_{b}^{a} F^{c M}{ }_{N},
$$

where $\tau_{c}$ here denotes a gauge generator in the adjoint representation.

Since the dimension of the 6 -vector representation of the Lorentz group is $d=6$, we have $\operatorname{Tr}_{V} I=6 N, \operatorname{Tr}_{V}(X)=-N R$, and so

$$
\begin{gathered}
\operatorname{Tr}_{V}\left(X^{2}\right)=N R_{M N} R^{M N}+4 C(A) F_{M N}^{a} F_{a}^{M N} \\
\operatorname{Tr}_{V}\left(Y_{M N} Y^{M N}\right)=-N R_{A B M N} R^{A B M N}-6 C(A) F_{M N}^{a} F_{a}^{M N},
\end{gathered}
$$

where $C(A)$ is the Dynkin index for $N$ fields in the adjoint representation: $\operatorname{tr}\left(\tau_{a} \tau_{b}\right)=$ $C(A) \delta_{a b}$. The subscript ' $V$ ' in these expressions is meant to emphasize that the trace has been taken over a vector field (as opposed to the physical spin-1 field, including ghosts).

These expressions suffice to compute $\operatorname{Tr}_{V}\left[a_{k}\right]$, for the vector field. Once specialized to Ricci flat geometries with vanishing background gauge field and constant scalars we find

$$
\begin{aligned}
& (-)^{F} \operatorname{Tr}_{V}\left[a_{0}\right]=6 N, \quad(-)^{F} \operatorname{Tr}_{V}\left[a_{1}\right]=0 \\
& (-)^{F} \operatorname{Tr}_{V}\left[a_{2}\right]=-\frac{N}{20} R_{A B M N} R^{A B M N} \\
& (-)^{F} \operatorname{Tr}_{V}\left[a_{3}\right]=\frac{5 N}{1512} I_{1}-\frac{N}{135} I_{2} .
\end{aligned}
$$


To this we must add the ghost contribution, which consists of $N$ complex scalar fields having fermionic statistics and transforming in the adjoint representation of the gauge group. The contributions to the $a_{k}$ may be read off from our previouslyquoted expressions for scalar fields in the special case $X=0$. For such fields we have

$$
\begin{aligned}
& (-)^{F} \operatorname{Tr}_{g h}\left[a_{0}\right]=-2 N, \quad(-)^{F} \operatorname{Tr}_{g h}\left[a_{1}\right]=0 \\
& (-)^{F} \operatorname{Tr}_{g h}\left[a_{2}\right]=-\frac{N}{90} R_{A B M N} R^{A B M N} \\
& (-)^{F} \operatorname{Tr}_{g h}\left[a_{3}\right]=\frac{17 N}{22680} I_{1}-\frac{N}{810} I_{2} .
\end{aligned}
$$

Summing the contributions of eqs. (3.22) and (3.23) gives the contribution of $N$ physical 6D massless gauge bosons:

$$
\begin{aligned}
& (-)^{F} \operatorname{Tr}_{1}\left[a_{0}\right]=4 N, \quad(-)^{F} \operatorname{Tr}_{1}\left[a_{1}\right]=0 \\
& (-)^{F} \operatorname{Tr}_{1}\left[a_{2}\right]=-\frac{11 N}{180} R_{A B M N} R^{A B M N} \\
& (-)^{F} \operatorname{Tr}_{1}\left[a_{3}\right]=\frac{23 N}{5670} I_{1}-\frac{7 N}{810} I_{2} .
\end{aligned}
$$

\section{2-Form Gauge Potentials}

A similar result for a 2-form gauge potential, $B_{M N}$, coupled only to the background metric may also be obtained, either by direct calculation [32] or by using results in the literature [40]. The starting point for this is the action

$$
S=-\frac{1}{12} \int d^{6} x \sqrt{-g} W_{3}(\varphi) G_{M N L} G^{M N L},
$$

where $G_{M N L}$ is the field strength, $G=\mathrm{d} B+\omega_{3}$ and $W_{3}(\varphi)$ is a known function of the background scalar fields.

In this case it is again possible to choose an appropriate gauge-averaging term and to keep track of all of the ghosts which result, along the lines as was done for the gauge potential above. Once summed over the results obtained in this way for ghosts and 2-form potentials, the result specialized to Ricci-flat geometries and constant scalar fields is given by

$$
\begin{aligned}
& (-)^{F} \operatorname{Tr}_{2 f}\left[a_{0}\right]=6 N, \quad(-)^{F} \operatorname{Tr}_{2 f}\left[a_{1}\right]=0, \\
& (-)^{F} \operatorname{Tr}_{2 f}\left[a_{2}\right]=\frac{11 N}{30} R_{A B M N} R^{A B M N}, \\
& (-)^{F} \operatorname{Tr}_{2 f}\left[a_{3}\right]=-\frac{1193 N}{7560} I_{1}+\frac{17 N}{54} I_{2},
\end{aligned}
$$

where $N$ counts the number of 2-form potentials which are present. 


\section{Gravitini}

We take spin-3/2 particles to be described by symplectic-Weyl vector-spinor fields, $\psi_{M}$, with a lagrangian given by

$$
\mathcal{L}_{V S}=-\frac{1}{2} \sqrt{-g} \bar{\psi}_{M} \Gamma^{M N P} D_{N} \psi_{P}
$$

where $\Gamma^{A B C}=\frac{1}{6}\left(\Gamma^{A} \Gamma^{B} \Gamma^{C}+\cdots\right)$ is the totally antisymmetric combination of gamma matrices. In order to put the spin-3/2 lagrangian into a form for which the general expressions for the Gilkey coefficients apply, it is convenient to use the gauge-averaging term

$$
\mathcal{L}_{g f}=\frac{1}{2} \sqrt{-g}(\overline{\Gamma \cdot \psi}) \not D(\Gamma \cdot \psi)
$$

With this term, and after making the field redefinition $\psi_{M} \rightarrow \psi_{M}-\frac{1}{4} \Gamma_{M} \Gamma \cdot \psi$, we find that the lagrangian simplifies to the following,

$$
\mathcal{L}_{V S}+\mathcal{L}_{g f}=-\frac{1}{2} \sqrt{-g} \bar{\psi}_{M} \not D \psi^{M}
$$

Thus, the one-loop contribution is

$$
i \Sigma_{3 / 2}=\frac{1}{2} \log \operatorname{det}\left[(\not D)_{B}^{A}\right]=\frac{1}{4} \log \operatorname{det}\left[\left(-\not D^{2}\right)_{B}^{A}\right] .
$$

For a vector-spinor the Lorentz generators are

$$
\left(J_{A B}\right)_{D}^{C}=-\frac{i}{2} \Gamma_{A B} \delta_{D}^{C}-i I\left(\delta_{A}^{C} \eta_{B D}-\delta_{B}^{C} \eta_{A D}\right),
$$

where $I$ is the $\mathcal{N} \times \mathcal{N}$ identity matrix, corresponding to the $\mathcal{N}=N d$ (unwritten) non-vector components of $\psi_{M}$. Using the identity $\not D^{2}=\square+\frac{1}{4}\left[\Gamma^{M}, \Gamma^{N}\right]\left[D_{M}, D_{N}\right]$, eq. (3.30) can be put into the required form, with $X$ given by

$$
X_{B}^{A}=\left(-\frac{1}{4} R+\frac{i}{2} F_{C D}^{a} \Gamma^{C D} t_{a}\right) \delta_{B}^{A}+\frac{1}{2} R_{B M N}^{A} \Gamma^{M N} .
$$

For simplicity of notation, we have suppressed writing the various identity matrices that appear in the above expression.

With the above expression for $X$, and remembering to multiply (as for the spin$1 / 2$ case) eq.(B.1) by an overall factor of $1 / 2$, we find for Ricci flat backgrounds

$$
\begin{aligned}
& (-)^{F} \operatorname{Tr}_{V S}\left[a_{0}\right]=-12 N, \quad(-)^{F} \operatorname{Tr}_{V S}\left[a_{1}\right]=0, \\
& (-)^{F} \operatorname{Tr}_{V S}\left[a_{2}\right]=-\frac{11 N}{40} R_{A B M N} R^{A B M N}, \\
& (-)^{F} \operatorname{Tr}_{V S}\left[a_{3}\right]=-\frac{113 N}{7560} I_{1}+\frac{17 N}{540} I_{2} .
\end{aligned}
$$


We next consider the contribution from the ghost fields. The gauge-averaging term eq. (3.28) introduces a complex, Faddeev-Popov spinor ghost as well as a Nielsen-Kallosh ghost, both of which must be summed over in order to obtain the result for the spin-3/2 particle. Since both types of ghosts have the same lagrangian as the massless spin-1/2 particle considered earlier, we must add to the vector-spinor result above $(-3)$ times the result for the spin-1/2 particle. Thus, for the gravitino we find

$$
\begin{aligned}
& (-)^{F} \operatorname{Tr}_{3 / 2}\left[a_{0}\right]=-6 N, \quad(-)^{F} \operatorname{Tr}_{3 / 2}\left[a_{1}\right]=0, \\
& (-)^{F} \operatorname{Tr}_{3 / 2}\left[a_{2}\right]=-\frac{73 N}{240} R_{A B M N} R^{A B M N} \\
& (-)^{F} \operatorname{Tr}_{3 / 2}\left[a_{3}\right]=-\frac{197 N}{15120} I_{1}+\frac{29 N}{1080} I_{2} .
\end{aligned}
$$

\section{Gravitons}

Finally, we turn to spin-2 particles. Although it is usually true that only a single spin-2 particle is massless in any given model, we include a parameter $N$ which counts the massive spin- 2 states. We do so because there is typically more than one massive spin-2 state in the models of interest, typically arising as part of a Kaluza-Klein tower or as excited string modes.

The lagrangian for a massless rank-two symmetric field is the Einstein-Hilbert action,

$$
\mathcal{L}_{E H}=-\frac{1}{2 \kappa^{2}} \sqrt{-g} R
$$

Writing the metric as $g_{M N}+h_{M N}$, we expand the action to quadratic order in the fluctuations, $h_{M N}$, about the background metric, $g_{M N}$. To obtain the required form, we use the gauge-fixing condition

$$
\mathcal{L}_{g f}=-\frac{1}{4 \kappa^{2}} \sqrt{-g}\left(D^{M} h_{M N}-\frac{1}{2} D_{N} h_{A}^{A}\right)^{2} .
$$

Finally, writing the resulting lagrangian in terms of the scalar, $\phi \equiv g_{M N} h^{M N}$, and the traceless symmetric tensor $\phi_{M N} \equiv h_{M N}-\frac{1}{6} g_{M N} \phi$, we find

$$
\begin{aligned}
& \frac{8 \kappa^{2}}{\sqrt{-g}}\left(\mathcal{L}_{E H}+\mathcal{L}_{g f}\right)=\phi^{M N}\left[\square \phi_{M N}+R \phi_{M N}-\left(\phi_{M A} R_{N}{ }^{A}+\phi_{N A} R_{M}{ }^{A}\right)\right. \\
& \left.-\left(R_{M A N B}+R_{M B N A}\right) \phi^{A B}\right]+\frac{2}{3} \phi^{M N} R_{M N} \phi-\frac{1}{3}\left(\phi \square \phi+\frac{1}{3} R \phi^{2}\right) .
\end{aligned}
$$

The fact that the scalar kinetic term has the wrong sign is well-known, and can be remedied by the field redefinition $\phi \rightarrow i \phi$. Notice also that, unlike in 4 dimensions, this procedure produces a cross-term between $\phi_{M N}$ and $\phi$. This term 


\begin{tabular}{|c|c|c|c|c|}
\hline Field & $(-)^{F} \operatorname{Tr}\left[a_{0}\right]$ & $(-)^{F} \operatorname{Tr}\left[a_{1}\right]$ & $(-)^{F} 720 \operatorname{Tr}\left[a_{2}\right]$ & $(-)^{F} 45360 \operatorname{Tr}\left[a_{3}\right]$ \\
\hline$\phi$ & 1 & 0 & $4 R_{A B M N}{ }^{2}$ & $-17 I_{1}+28 I_{2}$ \\
$\psi$ & -2 & 0 & $7 R_{A B M N^{2}}$ & $-29 I_{1}+70 I_{2}$ \\
$A_{M}$ & 4 & 0 & $-44 R_{A B M N^{2}}$ & $184 I_{1}-392 I_{2}$ \\
$A_{M N}$ & 6 & 0 & $264 R_{A B M N^{2}}$ & $-7158 I_{1}+14280 I_{2}$ \\
$\psi_{M}$ & -6 & 0 & $-219 R_{A B M N^{2}}$ & $-591 I_{1}+1218 I_{2}$ \\
$g_{M N}$ & 9 & 0 & $756 R_{A B M N}^{2}$ & $8919 I_{1}-17892 I_{2}$ \\
\hline
\end{tabular}

Table 1: Results for massless particles in 6 dimensions, including ghost contributions and specialized to Ricci-flat background metrics. Note that the boson fields in this table are real and the fermion fields are 6D symplectic-Weyl spinors.

will vanish for special choices of background metrics, and in particular for the Ricci flat case considered here. Specializing immediately to this situation, we see that the above lagrangian decouples into a rank-2 symmetric traceless piece and a scalar piece. However, because $R=0$, the results for the scalar fluctuations have already been calculated and are given by eq. (3.9). From eq. (3.37), we see that the rank-2 fluctuations have an $X$ given by

$$
X_{M N}^{A B}=R_{M N}^{A B}+R_{M N}^{B A},
$$

and so for $N$ rank-2 symmetric traceless fields, we find

$$
\begin{aligned}
& (-)^{F} \operatorname{Tr}_{\text {symtr }}\left[a_{0}\right]=20 N, \quad(-)^{F} \operatorname{Tr}_{\text {symtr }}\left[a_{1}\right]=0, \\
& (-)^{F} \operatorname{Tr}_{\text {symtr }}\left[a_{2}\right]=\frac{17 N}{18} R_{A B M N} R^{A B M N}, \\
& (-)^{F} \operatorname{Tr}_{\text {symtr }}\left[a_{3}\right]=\frac{2309 N}{11340} I_{1}-\frac{166 N}{405} I_{2} .
\end{aligned}
$$

Next, we consider the ghosts for this field. Since the gauge-averaging term is $f_{M}=D^{N} h_{N M}-\frac{1}{2} D_{M} h_{A}^{A}$, and since the gauge transformations are $\delta h_{M N}=$ $D_{M} \xi_{N}+D_{N} \xi_{M}$, we find the following transformation property

$$
\delta f_{M}=\square \xi_{M}-R_{M}^{A} \xi_{A},
$$

leading to a complex spin-one Faddeev-Popov fermionic ghost, $\omega_{M}$, with lagrangian

$$
\mathcal{L}=-\sqrt{-g} \omega_{M}^{*}\left(-\square \delta_{N}^{M}+R_{N}^{M}\right) \omega^{N}
$$

For the Ricci flat case, this has the same form as the vector lagrangian considered earlier. Thus, the complex ghost will contribute $(-2)$ times the result of a real vector 
field, eq. (3.22). Combining the contributions of the scalar and the Faddeev-Popov ghost to eq. (3.39), we obtain the following results for $N$ physical spin-2 particles,

$$
\begin{aligned}
& (-)^{F} \operatorname{Tr}_{2}\left[a_{0}\right]=9 N, \quad(-)^{F} \operatorname{Tr}_{2}\left[a_{1}\right]=0, \\
& (-)^{F} \operatorname{Tr}_{2}\left[a_{2}\right]=\frac{21 N}{20} R_{A B M N} R^{A B M N} \\
& (-)^{F} \operatorname{Tr}_{2}\left[a_{3}\right]=\frac{991 N}{5040} I_{1}-\frac{71 N}{180} I_{2} .
\end{aligned}
$$

The above results for the Gilkey coefficients for 6D massless particles are summarized in table (11).

\subsection{D Massive Particles}

It is relatively easy to compute the result for a massive particle in 6 dimensions using the above expressions for massless particles. We may do so because the massiveparticle formulae are obtainable by performing an appropriate sum over the corresponding massless-particle ones, by virtue of the higher-spin Higgs effect. We again specialize to vanishing background gauge fields and Ricci-flat metrics.

For example, for spin-1/2 particles an explicit 6D mass term necessarily relates a left- and right-handed spinor, and so the result for a massive spin-1/2 particle is obtained by summing the results for its constituent left- and right-handed components, $\psi_{ \pm}$. Since $a_{0}$ through $a_{3}$ are the same for both chiralities, this amounts to multiplying the Gilkey coefficients by a factor of 2 .

Similarly, for massive spin-1 particles it is convenient to use the covariant FujikawaLee-Sanda [30, 31] gauge-averaging term, $\xi^{-1}\left[D^{M} \mathcal{A}_{M}^{a}+c^{a}(\varphi)\right]^{2}$, with $c^{a}(\varphi)$ chosen to remove any scalar-vector cross terms of the schematic form $\mathcal{A}_{M} \partial^{M} \varphi$. In this case the gauge fields acquire a mass term which is identical to the mass term which results for the ghosts and for the would-be Goldstone bosons fields. We give explicit details of these calculations for arbitrary dimensions in a companion paper [32]. The upshot of all of this is that the Gikey-DeWitt coefficients for a massive spin-1 particle are obtained by summing the results for a massless spin-1 particle with the result for a real spinless particle with $X=0$, leading to

$$
\begin{aligned}
& (-)^{F} \operatorname{Tr}_{1 m}\left[a_{0}\right]=5 N, \quad(-)^{F} \operatorname{Tr}_{1 m}\left[a_{1}\right]=0 \\
& (-)^{F} \operatorname{Tr}_{1 m}\left[a_{2}\right]=-\frac{10 N}{180} R_{A B M N} R^{A B M N} \\
& (-)^{F} \operatorname{Tr}_{1 m}\left[a_{3}\right]=\frac{167 N}{45360} I_{1}-\frac{13 N}{1620} I_{2} .
\end{aligned}
$$

A similar argument applies for other higher-spin fields, with the result being to 


\begin{tabular}{|c|c|c|c|c|}
\hline Multiplet & $(-)^{F} \operatorname{Tr}\left[a_{0}\right]$ & $(-)^{F} \operatorname{Tr}\left[a_{1}\right]$ & $(-)^{F} 360 \operatorname{Tr}\left[a_{2}\right]$ & $(-)^{F} 45360 \operatorname{Tr}\left[a_{3}\right]$ \\
\hline$\phi$ & 1 & 0 & $2 R_{A B M N}{ }^{2}$ & $-17 I_{1}+28 I_{2}$ \\
$\psi$ & -4 & 0 & $7 R_{A B M N}{ }^{2}$ & $-58 I_{1}+140 I_{2}$ \\
$A_{M}$ & 5 & 0 & $-20 R_{A B M N}{ }^{2}$ & $167 I_{1}-364 I_{2}$ \\
$A_{M N}$ & 10 & 0 & $110 R_{A B M N}{ }^{2}$ & $-6974 I_{1}+13888 I_{2}$ \\
$\psi_{M}$ & -16 & 0 & $-212 R_{A B M N}{ }^{2}$ & $-1240 I_{1}+2576 I_{2}$ \\
$g_{M N}$ & 14 & 0 & $358 R_{A B M N}^{2}$ & $9086 I_{1}-18256 I_{2}$ \\
\hline
\end{tabular}

Table 2: Results for massive particles in 6 dimensions, specialized to Ricci-flat backgrounds. Note that in this table the bosonic fields are real and the spinors are symplectic, but not Weyl.

sum the lower-spin formulae in the following schematic combinations [32]:

$$
\begin{aligned}
\phi^{m} & \rightarrow \phi \\
\psi^{m} & \rightarrow \psi_{+}+\psi_{-} \\
A_{M}^{m} & \rightarrow A_{M}+\phi \\
A_{M N}^{m} & \rightarrow A_{M N}+A_{M} \\
\psi_{M}^{m} & \rightarrow \psi_{M+}+\psi_{M-}+\psi_{+}+\psi_{-} \\
g_{M N}^{m} & \rightarrow g_{M N}+A_{M}+\phi .
\end{aligned}
$$

In writing these relations we use that the dimension of spacetime of interest here is even, so that Weyl spinors, $\psi_{ \pm}$, may be defined.

A check on these relations can also be obtained in an alternative way. Recall that, for flat space, the little group which preserves the momentum of a massive particle in $n$ dimensions is $S O(n-1)$, while that which preserves the standard null momentum of a massless particle is $S O(n-2)$. For the purposes of calculating ultraviolet divergences, the counting of states for massive particles in $n$ dimensions is equivalent to the counting for massless particles in $n+1$ dimensions. Using the more general results for the Gilkey coefficients of higher spins in $n$ dimensions of ref. [32, 40], it can be shown that the above counting of massive states agrees with the result for massless fields in $n+1$ dimensions. Table (2) summarizes the results which these arguments imply for massive particles in 6 dimensions.

\subsection{Supersymmetric Multiplets}

In supergravity theories the ultraviolet sensitivity of the low-energy theory is often weaker than in non-supersymmetric models. This weaker sensitivity arises due to the tendency of bosons and fermions to cancel in loops provided their masses and 
couplings are equal. These cancellations may be seen by summing the above results over the particles appearing in the appropriate supermultiplets.

The result for the ultraviolet-sensitive part of the one-loop action obtained by integrating out a supermultiplet is given by

$$
\Sigma=\frac{1}{2}\left(\frac{1}{4 \pi}\right)^{n / 2} \int d^{n} x \sqrt{-g} \sum_{p}(-)^{F(p)} \sum_{k=0}^{n / 2} \Gamma(k-n / 2) \operatorname{Tr}_{p}\left[m_{p}^{n-2 k} a_{k}\right]
$$

where the sum on $p$ runs over the elements of a supermultiplet. As is clear from this expression, it is weighted sums of the form $\sum_{p}(-)^{F(p)} \operatorname{Tr}_{p}\left[m_{p}^{n-2 k} a_{k}\right]$ which control the UV sensitivity of supersymmetric theories. For example the equal numbers of bose and fermi states within a supermultiplet automatically imply

$$
\sum_{p \in \mathrm{sm}}(-)^{F(p)} \operatorname{Tr}_{p}\left[a_{0}\right]=0
$$

which expresses the usual cancellation of the contributions of bosons and fermions to the cosmological constant if all of the elements of a supermultiplet share the same mass.

The next few sections record the analogous result for the other coefficients $a_{1}$ through $a_{3}$ - once summed over the particles within various $6 \mathrm{D}$ supermultiplets, under the assumption that all elements of the supermultiplet have the same $6 \mathrm{D}$ mass. Notice that our discussion of the equivalence between performing the sum over the UV sensitive part of the 4D contribution of each KK mode and the UV sensitivity as computed using the full $6 \mathrm{D}$ fields shows that the assumption of equal masses within a $6 \mathrm{D}$ supermultiplet relies only on there being unbroken $(2,0)$ supersymmetry at the high energy scale, $M$, appropriate to the compactification from higher dimensions down to $6 \mathrm{D}$. Provided that $M \gg m_{\mathrm{KK}} \sim 1 / r$, this assumption does not require that some supersymmetry remains unbroken below the scale of the KK masses encountered in the compactification from $6 \mathrm{D}$ down to $4 \mathrm{D}$.

\section{Massless 6D Supermultiplets}

We first summarize the particle content of the simplest 6D supermultiplets, start-

ing first with massless multiplets and then moving on to massive multiplets. Our discussion follows that of ref. [33].

Massless multiplets are partially characterized by their representation properties for the 'little group' which preserves the form of a standard light-like energymomentum vector. In $5+1$ dimensions, the light-like little group contains the rotations, $S O(4)$, of the 4 spatial dimensions transverse to the direction of motion of the standard light-like momentum. The representations of $S O(4) \sim S U(2) \times S U(2)$ 


\begin{tabular}{|c|c|c|}
\hline Multiplet & Representation & Field Equivalent \\
\hline $\mathbf{4}$ & $2^{2}=(\mathbf{2}, \mathbf{1} ; \mathbf{1})+(\mathbf{1}, \mathbf{1} ; \mathbf{2})$ & $(\psi, 2 \phi)$ \\
$\mathbf{8}$ & $(\mathbf{1}, \mathbf{2} ; \mathbf{1}) \times 2^{2}=(\mathbf{2}, \mathbf{2} ; \mathbf{1})+(\mathbf{1}, \mathbf{2} ; \mathbf{2})$ & $\left(A_{M}, 2 \psi\right)$ \\
$\mathbf{8}^{\prime}$ & $(\mathbf{2}, \mathbf{1} ; \mathbf{1}) \times 2^{2}=(\mathbf{3}, \mathbf{1} ; \mathbf{1})+(\mathbf{1}, \mathbf{1} ; \mathbf{1})+(\mathbf{2}, \mathbf{1} ; \mathbf{2})$ & $\left(A_{M N}, 2 \psi, \phi\right)$ \\
$\mathbf{1 2}$ & $(\mathbf{1}, \mathbf{3} ; \mathbf{1}) \times 2^{2}=(\mathbf{2}, \mathbf{3} ; \mathbf{1})+(\mathbf{1}, \mathbf{3} ; \mathbf{2})$ & $\left(\psi_{M}, 2 A_{M N}\right)$ \\
$\mathbf{1 6}$ & $(\mathbf{2}, \mathbf{2} ; \mathbf{1}) \times 2^{2}=(\mathbf{3}, \mathbf{2} ; \mathbf{1})+(\mathbf{1}, \mathbf{2} ; \mathbf{1})+(\mathbf{2}, \mathbf{2} ; \mathbf{2})$ & $\left(\psi_{M}, 2 A_{M}, \psi\right)$ \\
$\mathbf{2 4}$ & $(\mathbf{2 , 3} ; \mathbf{1}) \times 2^{2}=(\mathbf{3}, \mathbf{3} ; \mathbf{1})+(\mathbf{1}, \mathbf{3} ; \mathbf{1})+(\mathbf{2 , 3} \mathbf{3})$ & $\left(g_{M N}, 2 \psi_{M}, A_{M N}\right)$ \\
\hline
\end{tabular}

Table 3: Massless representations of $(2,0)$ supersymmetry in 6 dimensions, labelled by the dimension of the representation of the corresponding little-group algebra, and by the corresponding $6 \mathrm{D}$ field content. The fermions are taken to be symplectic-Weyl, and the 2 -form potentials are similarly self (or anti-self) dual.

corresponding to the simplest particle types are

$$
(\mathbf{1}, \mathbf{1}): \operatorname{scalar}(\phi)
$$

$(\mathbf{2}, \mathbf{1}),(\mathbf{1}, \mathbf{2})$ : Weyl spinors $\left(\psi_{ \pm}\right)$

$(\mathbf{2}, \mathbf{2})$ : gauge potential $\left(A_{M}\right)$

$(\mathbf{3}, \mathbf{1}),(\mathbf{1}, \mathbf{3})$ : (anti) self-dual 2-form potentials $\left(A_{M N}^{ \pm}\right)$

$(\mathbf{3}, \mathbf{2}),(\mathbf{2}, \mathbf{3})$ : Weyl gravitino $\left(\psi_{M}^{ \pm}\right)$

$(\mathbf{3}, \mathbf{3})$ : graviton $\left(g_{M N}\right)$

where we denote the particle type by the field with which it is usually represented. The two representations listed for the Weyl fermions correspond to the two types of chiralities these fermions can have. Similarly, the two representations listed for the 2 -form potential correspond to the self- and anti-self-dual pieces, which are defined to satisfy $G_{M N P}= \pm \epsilon_{M N P Q R S} G^{Q R S}$.

Massless supermultiplets are also characterized by the action of the graded automorphism symmetry which is generated by the supercharges, which transform as spinors of $S O(4)$. Since the fundamental spinor representations $-\mathbf{2}_{+}=(\mathbf{2}, \mathbf{1})$ and $\mathbf{2}_{-}=(\mathbf{1}, \mathbf{2})$ - of $S O(4) \sim S U(2) \times S U(2)$ are pseudo-real, the graded automorphism group in 6 dimensions is $U S p\left(N_{+}\right) \times U S p\left(N_{-}\right)$, where $N_{+}$and $N_{-}$(which must both be even) characterize the number of independent, pseudo-real chiral supersymmetries. The minimal supersymmetry algebra therefore corresponds to $\left(N_{+}, N_{-}\right)=$ $(2,0)$, and so $N=N_{+}+N_{-}=2$ [33]. The relevant little group characterizing the massless supermultiplets is then $G=S O(4) \times U S p(2) \sim S U(2) \times S U(2) \times U S p(2)$, under which the active supercharges transform in the representation $(\mathbf{2}, \mathbf{1} ; \mathbf{2})$.

The minimal representation of this little algebra has dimension $2^{2}=4$, and 


\begin{tabular}{|c|c|c|c|c|}
\hline Multiplet & $(-)^{F} \operatorname{Tr}\left[a_{0}\right]$ & $(-)^{F} \operatorname{Tr}\left[a_{1}\right]$ & $(-)^{F} 48 \operatorname{Tr}\left[a_{2}\right]$ & $(-)^{F} 720 \operatorname{Tr}\left[a_{3}\right]$ \\
\hline $\mathbf{4}$ & 0 & 0 & $R_{A B M N}{ }^{2}$ & $\left(-I_{1}+2 I_{2}\right)$ \\
$\mathbf{8}$ & 0 & 0 & $-2 R_{A B M N}{ }^{2}$ & $-2\left(-I_{1}+2 I_{2}\right)$ \\
$\mathbf{8}^{\prime}$ & 0 & 0 & $10 R_{A B M N}{ }^{2}$ & $58\left(-I_{1}+2 I_{2}\right)$ \\
$\mathbf{1 2}$ & 0 & 0 & $3 R_{A B M N}{ }^{2}$ & $123\left(-I_{1}+2 I_{2}\right)$ \\
$\mathbf{1 6}$ & 0 & 0 & $-20 R_{A B M N}{ }^{2}$ & $4\left(-I_{1}+2 I_{2}\right)$ \\
$\mathbf{2 4}$ & 0 & 0 & $30 R_{A B M N}{ }^{2}$ & $-66\left(-I_{1}+2 I_{2}\right)$ \\
\hline
\end{tabular}

Table 4: Results for the statistics-weighted sum over Gilkey coefficients for massless 6D supermultiplets, specialized to vanishing gauge fluxes and Ricci-flat backgrounds.

transforms under $G$ like [33]

$$
4=(2,1 ; 1)+(1,1 ; 2)
$$

This consists of 2 real (1 complex) scalars and a single symplectic-Weyl fermion, and so consists of 2 bosonic and 2 fermionic states.

Higher-dimensional representations may be obtained from this minimal one by taking direct products of it with an irreducible representation of the bosonic part of the little group. Table (3) lists some possible representations which are obtained in this way, including the hyper-multiplet (4), gauge multiplet (8), tensor multiplet $\left(\mathbf{8}^{\prime}\right)$, two types of gravitino multiplet $(\mathbf{1 2}, \mathbf{1 6})$ and the graviton multiplet $(\mathbf{2 4})$. To derive the results in this table, we use the standard results for $S U(2): \mathbf{2} \times \mathbf{2}=\mathbf{1}+\mathbf{3}$, $2 \times 3=2+4$ and $2 \times 4=3+5$.

We may now sum the previous expressions for the Gilkey coefficients over the particle content of these massless 6D supermultiplets. The results obtained for $\operatorname{Tr}_{\mathrm{sm}}\left[a_{k}\right]=\sum_{p \in \mathrm{sm}}(-)^{F(p)} \operatorname{Tr}_{p}\left[a_{k}\right]$ if the particles all share the same mass are summarized in table 1 . Notice that the resulting expressions for $\operatorname{Tr}_{\mathrm{sm}}\left[a_{3}\right]$ are proportional to the combination $-I_{1}+2 I_{2}$ which gives the Euler number density for compact $6 \mathrm{D}$ manifolds.

\section{Massive 6D Supermultiplets}

The massive representations of $(2,0) 6 \mathrm{D}$ supersymmetry are found in a similar manner, except in this case the little group for the time-like energy-momentum vector appropriate to massive fields is $S O(5)$. The particle types and fields corresponding 


\begin{tabular}{|c|c|c|c|}
\hline Multiplet & \multicolumn{2}{|r|}{ Representation } & Field Equivalent \\
\hline $16_{m}$ & 2 & $=(5,1)+(1,3)+(4,2)$ & $\left(A_{M}^{m}, 2 \psi^{m}, 3 \phi^{m}\right)$ \\
\hline $64_{m}$ & $(4,1) \times 2$ & $\begin{aligned}= & (16,1)+(4,1)+(4,3)+ \\
& (10,2)+(5,2)+(1,2)\end{aligned}$ & $\left(\psi_{M}^{m}, 2 A_{M N}^{m}, 2 A_{M}^{m}, 4 \psi^{m}, 2 \phi^{m}\right)$ \\
\hline $80_{m}$ & $(\mathbf{5}, \mathbf{1}) \times 2$ & $\begin{aligned}= & (14,1)+(10,1)+(1,1)+ \\
& (5,3)+(16,2)+(4,2)\end{aligned}$ & $\left(g_{M N}^{m}, 2 \psi_{M}^{m}, A_{M N}^{m}, 3 A_{M}^{m}, 2 \psi^{m}, \phi^{m}\right)$ \\
\hline
\end{tabular}

Table 5: Massive representations of $(2,0)$ supersymmetry in 6 dimensions, labelled by their dimension. Note that the fermions are not chiral and the 2 -form potentials are not self-dual or antiself-dual.

to the representations of $S O(5)$ are as follows:

$$
\begin{aligned}
1 \text { : } & \text { massive scalar }\left(\phi^{m}\right) \\
4: & \text { massive spinor }\left(\psi^{m}\right) \\
5: & \text { massive gauge potential }\left(A_{M}^{m}\right) \\
10: & \text { massive 2-form }\left(A_{M N}^{m}\right) \\
16: & \text { massive gravitino }\left(\psi_{M}^{m}\right) \\
14: & \text { massive graviton }\left(g_{M N}^{m}\right) .
\end{aligned}
$$

The little algebra for the supersymmetry representations is therefore $S O(5) \times$ $U S p(2)$. The irreducible spinor representations of $S O(5)$ are not chiral, and are 4dimensional, and so the number of supercharges doubles in going from light-like to time-like representations. ${ }^{9}$ It follows that the dimensionality of the minimal representation is the square of what it was in the light-like situation: $2^{4}=16$, with the following decomposition under $S O(5) \times U S p(2)$ [33]:

$$
16_{m}=(5,1)+(1,3)+(4,2) .
$$

Again, we find all other representations by taking appropriate direct products. To do so, we use the following standard results for $S O(5): \mathbf{4} \times \mathbf{4}=\mathbf{1 0}+\mathbf{5}+\mathbf{1}$ and $4 \times \mathbf{5}=\mathbf{1 6}+\mathbf{4}$, leading to the massive supermultiplets given in table (5). Since it is possible to understand massive particles in terms of combinations of massless ones, the same is true for massive supermultiplets. Using these results of eq. (3.44), we find the unique decomposition of the massive multiplets in terms of the massless ones:

$$
\begin{aligned}
& \mathbf{1 6}_{m} \rightarrow 2(\mathbf{4})+\mathbf{8} \\
& \mathbf{6 4}_{m} \rightarrow \mathbf{4}+2(\mathbf{8})+2\left(\mathbf{8}^{\prime}\right)+\mathbf{1 2}+\mathbf{1 6} \\
& \mathbf{8 0}_{m} \rightarrow 2(\mathbf{4})+\mathbf{8}+\mathbf{8}^{\prime}+2(\mathbf{1 6})+\mathbf{2 4} .
\end{aligned}
$$

\footnotetext{
${ }^{9} \mathrm{We}$ assume here vanishing central charges - and so no 'short' multiplets.
} 


\begin{tabular}{|c|c|c|c|c|}
\hline Multiplet & $(-)^{F} \operatorname{Tr}\left[a_{0}\right]$ & $(-)^{F} \operatorname{Tr}\left[a_{1}\right]$ & $(-)^{F} \operatorname{Tr}\left[a_{2}\right]$ & $(-)^{F} \operatorname{Tr}\left[a_{3}\right]$ \\
\hline $\mathbf{1 6}_{m}$ & 0 & 0 & 0 & 0 \\
$\mathbf{6 4}_{m}$ & 0 & 0 & 0 & $\frac{1}{3}\left(-I_{1}+2 I_{2}\right)$ \\
$\mathbf{8 0}_{m}$ & 0 & 0 & 0 & 0 \\
\hline
\end{tabular}

Table 6: Results for massive 6D multiplets (Ricci flat).

Labelling the multiplets as in tables (3) and (5), it is straightforward to compute the results for the statistics-weighted sum of the Gilkey coefficients over the massive supermultiplet particle content. The results obtained in this way are summarized in Table 6. What is striking about this table is the vanishing of the contributions to $a_{0}$ through $a_{2}$, which ensures the absence within the effective action of positive powers of the common $6 \mathrm{D}$ mass $M$ of a supermultiplet of degenerate massive particles.

\subsection{Higher-Dimensional Field Content}

Of particular interest are those massive 6D supermultiplets which are obtained by dimensionally reducing the various $10 \mathrm{D}$ supergravities to six dimensions. The simplest such compactifications are obtained by reducing the higher-dimensional theories on a 4-torus. Since the IIA theory can be obtained from 11-dimensional supergravity via dimensional reduction on $S^{1}$, the results we obtain for the Type IIA theory dimensionally reduced on a 4 -torus are equivalent to what is obtained from 11-dimensional supergravity dimensionally reduced on a 5-torus.

For the purposes of the present argument all that matters is the total number of each type of massive $6 \mathrm{D}$ fields which are produced by such a reduction. Our purpose in this section is to show that the massive field content which is obtained by such a dimensional reduction is the same as would be obtained by combining a small number of the massive $\mathbf{1 6}_{m}, \mathbf{6 4}_{m}$ and $\mathbf{8 0}_{m}$ representations of $(2,0) 6 \mathrm{D}$ supersymmetry. ${ }^{10}$ Since we know that each of these multiplets gives a vanishing contributions to the first 3 heat-kernel coefficients, the same must also be true of the contributions of the massive $6 \mathrm{D}$ states which are obtained by dimensional reduction.

\section{Type IIA and Type IIB Supergravities}

The field content of Type IIA supergravity in ten dimensions consists of: a graviton; a 3-form, 2-form, 1-form, and 0-form; two majorana-Weyl gravitini having opposite $10 \mathrm{D}$ chiralities; and two majorana-Weyl dilatini having opposite 10D chiralities. The

\footnotetext{
${ }^{10}$ This need not imply that these massive states actually transform in these representations under $(2,0)$ supersymmetry, such as because of the possible presence of central charges and short multiplets.
} 
IIB theory is obtainable from the IIA theory by giving all fermions the same chirality, and by trading the 3-form and the 1-form for a self-dual 4-form, a 2-form, and a 0form.

Since each majorana-Weyl spinor in 10D has 16 real components, reduction on a torus (in the absence of any Scherk-Schwartz supersymmetry-breaking twists [34]) gives $(8,8) 6 \mathrm{D}$ supersymmetry. The massless sector of the resulting $6 \mathrm{D}$ theory can therefore be described in terms of $(2,0)$ supersymmetry multiplets, and arranges itself into the following collection of massless $(2,0)$ supermultipets,

$$
\text { Type IIA/B : } \mathbf{2 4}+4(\mathbf{1 6})+2(\mathbf{1 2})+5\left(\mathbf{8}^{\prime}\right)+8(\mathbf{8})+10(\mathbf{4}) \text {, }
$$

with both Type IIA and IIB supergravities giving the same multiplet content when dimensionally reduced on a 4-torus. In deriving these results, we use the equivalence (in 6 dimensions) of a 3 -form and a 1-form gauge potential.

Using the results from table (4), we find the following statistics-weighted sum for the Gilkey coefficients produced by the massless sector of the 6D theory:

$$
\begin{aligned}
& \operatorname{Tr}\left[a_{0}\right]=\operatorname{Tr}\left[a_{1}\right]=\operatorname{Tr}\left[a_{2}\right]=0, \\
& \operatorname{Tr}\left[a_{3}\right]=\frac{2}{3}\left(-I_{1}+2 I_{2}\right) .
\end{aligned}
$$

The only UV-sensitive quantity is a topological term proportional to the Eulernumber density. If we had not used the duality relationship to exchange the 3 -form potential for a 1-form potential, even the coefficient of the Euler-number term would have vanished, because in 6 dimensions $40,{ }^{11}$

$$
\operatorname{Tr}_{3 f}\left[a_{3}\right]-\operatorname{Tr}_{1 f}\left[a_{3}\right]=\frac{2}{3}\left(-I_{1}+2 I_{2}\right) .
$$

A similar statement can be made for the massive KK modes produced by any such $(8,8)$-supersymmetric dimensional reduction on a 4 -torus. In this case the massive states have the same field content as do the following $6 \mathrm{D}$ massive $(2,0)$ supersymmetry representations:

$$
\text { Type IIA/B : } \mathbf{8 0}_{m}+2\left(\mathbf{6 4}_{m}\right)+3\left(\mathbf{1 6}_{m}\right) \text {, }
$$

The vanishing of $\operatorname{Tr}\left[a_{0}\right]$ through $\operatorname{Tr}\left[a_{2}\right]$ for the massive multiplets ensures the vanishing of any UV sensitive contributions from the KK modes obtained when reducing from 10 to 6 dimensions (provided these are not in short multiplets).

\footnotetext{
${ }^{11}$ This is an example of the breakdown of naive equivalences between different field representation descriptions of the same particle [15].
} 


\section{Type I and Heterotic Theories}

Type I and heterotic theories consist of a 10D, $N=1$, supergravity multiplet coupled to a 10D Yang-Mills multiplet. The relevant gauge group is $S O(32)$ for the type I and $\operatorname{Spin}(32) / Z_{2}$ for the heterotic A theory, and $E_{8} \times E_{8}$ for the heterotic B theory. The supergravity multiplet contains a graviton, a 2-form gauge potential, a scalar dilaton, and a majorana-Weyl gravitino and a majorana-Weyl spin- $1 / 2$ fermion, both having the same chirality. The gauge multiplet contains $N_{A}$ gauge fields and $N_{A}$ spin-1/2 fermions, where $N_{A}=496$ is the dimension of the gauge group.

Dimensionally reducing on a 4 -torus to six dimensions without breaking any supersymmetries in this case ensures unbroken $(4,4) 6 \mathrm{D}$ supersymmetry, with the massless sector arranging itself into the following $(2,0)$ supermultiplets (where we keep separate the supergravity and the Yang-Mills fields):

$$
\begin{aligned}
\text { Sugra }: & \mathbf{2 4}+2(\mathbf{1 6})+\left(\mathbf{8}^{\prime}\right)+4(\mathbf{8})+8(\mathbf{4}) \\
\mathrm{YM}: & N_{A}(\mathbf{8})+2 N_{A}(\mathbf{4}) .
\end{aligned}
$$

Using the results from table (4), we find for the massless sectors of both the supergravity and the Yang-Mills theory

$$
\operatorname{Tr}\left[a_{0}\right]=\operatorname{Tr}\left[a_{1}\right]=\operatorname{Tr}\left[a_{2}\right]=\operatorname{Tr}\left[a_{3}\right]=0 .
$$

We again find that the dimensionally reduced theory shares the UV properties of its higher-dimensional counterpart [40].

The contributions of massive KK modes may similarly be analyzed in this case, with the result that there is a vanishing contribution to $\operatorname{Tr}\left[a_{0}\right], \operatorname{Tr}\left[a_{1}\right]$ and $\operatorname{Tr}\left[a_{2}\right]$.

\section{Acknowledgements}

We thank Y. Aghababaie, Z. Chacko, J. Elliot, G. Gabadadze, D. Ghilencia, A. Tseytlin and F. Quevedo for helpful discussions about heat-kernel methods and 6D Casimir energies. C.B.'s research is supported by a grant from NSERC (Canada).

\section{A. Dimensional Regularization and Cutoffs}

In this paper all loops are computed using dimensional regularization since this is by far the most convenient for practical calculations. At first sight this might appear to limit our discussion of ultraviolet sensitivity, since this is traditionally treated in terms of quadratic, quartic and higher ultraviolet divergences, which are absent in dimensional regularization. This is a special case of the more general question of whether (and how) dimensional regularization can be reconciled with effective 
field theory techniques, which are usually phrased in terms of cutoffs, through the integrating out high-energy modes.

The resolution of this apparent difficulty with dimensional regularization has been known for quite some time, however, both in the general context of effective lagrangians [28, 29, 41] and for explicit examples of UV-sensitivity [42]. The result is that all of the usual UV-sensitivity issues may also be addressed using dimensional regularization, and indeed arise there within what is arguably a more physical context. In this section we briefly recap these issues, following the discussion of refs. [42], in which the same issues arise within another context.

The issue of UV-sensitivity arises when a physical observable, $\mathcal{O}$, depends on two very different physical mass scales, say $m$ and $M$ with $m \ll M$. (The dependence on other parameters, like couplings etc. are suppressed in what follows.) For instance, $M$ and $m$ might be the masses of two types of particles, which are themselves physically measurable, at least in principle. It is typically true that the expression for $\mathcal{O}(m, M)$ simplifies dramatically when $m \ll M$, due to the ability of expanding the result in powers of the small ratio $m / M$

$$
\mathcal{O}(m, M)=m^{d}\left[C_{n}\left(\frac{m}{M}\right)^{n}+C_{n-1}\left(\frac{m}{M}\right)^{n-1}+\cdots\right],
$$

where $d$ is the mass dimension of $\mathcal{O}$ and the coefficients $C_{k}$ might also depend logarithmically on $M / m$. For the vast majority of observables the starting power, $n$, satisfies $n>0$, and so the dominant dependence on the large scale, $M$, has the form $(m / M)^{n} \log ^{p}(m / M)$ (for some $p \geq 0$ ) and so vanishes as $m / M \rightarrow 0$ (with the logarithmic dependence arising through the coefficient, $\left.C_{n}\right)$. UV-sensitivity corresponds to the case where $n \leq 0$, in which case it can be singular to take the limit $M \rightarrow \infty$ in $\mathcal{O}$ with all other scales fixed.

Cutoffs arise if the large hierarchy, $M / m$, is exploited to integrate out all modes having energies $E>\Lambda$ to obtain an effective theory containing only light particles which is applicable at scales $E<\Lambda$. Nothing physical depends on the scale $\Lambda$ in such a construction because it is simply a book-keeping tool which organizes how calculations are done. This independence happens in detail through cancellations between the $\Lambda$-dependence of the effective interactions contained in the low-energy lagrangian, $\mathcal{L}$, and the dependence on $\Lambda$ which arises as cutoffs within loop integrals within the low-energy theory. (See, for instance, refs. 28, 29] for details of this cancellation.)

Within an effective field theory potential confusion can arise between the dependence of observables, like $\mathcal{O}$, on physical heavy masses, like $M$, on the one hand, and the dependence of the low-energy contribution to $\mathcal{O}$, on the cutoff $\Lambda$ on the other. For instance, if a low-energy calculation of $\mathcal{O}$ has a low-energy contribution 
$\mathcal{O}_{\text {le }} \sim A \Lambda^{n}$, it is tempting to draw the conclusion that the final result, $\mathcal{O}$, depends on $M$ like $\mathcal{O} \sim \hat{A} M^{n}$, for $\hat{A} \sim A$.

This conclusion actually works quite well for logarithmic UV-sensitivity, because in that case the low energy result, $\mathcal{O}_{\text {le }}=A \log (\Lambda / m)$, must combine with a highenergy (effective coupling) contribution, $\mathcal{O}_{\text {he }}=A^{\prime} \log (M / \Lambda)$, to give a $\Lambda$-independent result. For logarithms this dictates that $A=A^{\prime}=\hat{A}$, so that

$$
\mathcal{O}=A \log \left(\frac{\Lambda}{m}\right)+A \log \left(\frac{M}{\Lambda}\right)=A \log \left(\frac{M}{m}\right),
$$

and so the coefficient, $A$, of the physical large logarithm, $\log (M / m)$, may be computed using only the coefficient of the divergence, $\log (\Lambda / m)$, in the low-energy theory.

The argument can fail, however, for power divergences, for which a low-energy contribution, $\mathcal{O}_{\text {le }}=A \Lambda^{2}$, can cancel with a high-energy coefficient, $\mathcal{O}_{\text {he }}=B M^{2}-$ $A \Lambda^{2}$, to give the physical answer, $\mathcal{O}=B M^{2}$. In this case the coefficient, $B$, of $M^{2}$ in the full result need not be related at all to the coefficient, $A$, of the divergence $\Lambda^{2}$ within the low-energy theory.

Dimensional regularization and a modified form of modified-minimal subtraction has many calculational advantages, including the simplicity of never introducing an unphysical scale like $\Lambda$ as a power during intermediate steps of a calculation. This does not mean that UV sensitivity cannot arise within a dimensionally-regulated theory, of course. It merely means that it arises in a more explicit way - through the matching conditions which must be applied at a physical threshhold, $\mu=M$, when one renormalizes the action through this scale and removes the heavy particles having mass $M$ from the theory [41]. Within this language dangerous powers of $M$ are only obtained when physics at scale $M$ is integrated out in this way, through explicit contact with the physics of this scale.

In the body of the text we formulate the UV-sensitivity of the effective $4 \mathrm{D}$ cosmological constant in this way, using dimensional methods to regularize divergent integrations. And it is because of this choice that we find contributions of order $M^{4}$ when integrating out particles of mass $M$, rather than following the $\Lambda$-dependence in a cutoff regularization.

\section{B. The Heat Kernel Coefficients}

In this appendix we collect for convenience the explicit expressions for the coefficients $a_{0}$ through $a_{3}$ in their general form for manifolds without singularities and boundaries. These are known for general background metrics, $g_{M N}$, and for general 
$X$ and $Y_{M N}=\left[D_{M}, D_{N}\right]$. The first few are given explicitly by $[13,16]:{ }^{12}$

$$
\begin{aligned}
a_{0}= & I \\
a_{1}= & -\frac{1}{6}(R I+6 X) \\
a_{2}= & \frac{1}{360}\left(2 R_{A B M N} R^{A B M N}-2 R_{M N} R^{M N}+5 R^{2}-12 \square R\right) I \\
& +\frac{1}{6} R X+\frac{1}{2} X^{2}-\frac{1}{6} \square X+\frac{1}{12} Y_{M N} Y^{M N} \\
a_{3}= & \frac{1}{7 !}\left(-18 \square^{2} R+17 D_{M} R D^{M} R-2 D_{L} R_{M N} D^{L} R^{M N}-4 D_{L} R_{M N} D^{N} R^{M L}\right. \\
& +9 D_{K} R_{M N L P} D^{K} R^{M N L P}+28 R \square R-8 R_{M N} \square R^{M N}+24 R^{M}{ }_{N} D^{L} D^{N} R_{M L} \\
& +12 R_{M N L P} \square R^{M N L P}-\frac{35}{9} R^{3}+\frac{14}{3} R R_{M N} R^{M N}-\frac{14}{3} R R_{A B M N} R^{A B M N} \\
& +\frac{208}{9} R^{M}{ }_{N} R_{M L} R^{N L}-\frac{64}{3} R^{M N} R^{K L} R_{M K N L}+\frac{16}{3} R^{M}{ }_{N} R_{M K L P} R^{N K L P} \\
& \left.-\frac{44}{9} R^{A B}{ }_{M N} R_{A B K L} R^{M N K L}-\frac{80}{9} R_{B}^{A}{ }_{N} R_{A K M P} R^{B K N P}\right) I \\
& +\frac{1}{360}\left(8 D_{M} Y_{N K} D^{M} Y^{N K}+2 D^{M} Y_{N M} D_{K} Y^{N K}+12 Y^{M N} \square Y_{M N}\right. \\
& -12 Y_{N}^{M} Y_{K}^{N} Y_{M}^{K}{ }_{M}-6 R^{M N K L} Y_{M N} Y_{K L}+4 R^{M}{ }_{N} Y_{M K} Y^{N K} \\
& -5 R Y_{M N} Y_{M N}-6 \square{ }^{2} X+60 X \square X+30 D_{M} X D^{M} X-60 X^{3} \\
& -30 X Y_{M N} Y_{M N}+10 R \square X+4 R^{M N} D_{M} D_{N} X+12 D^{M} R D_{M} X-30 X^{2} R \\
& \left.+12 X \square R-5 X R^{2}+2 X R_{M N} R^{M N}-2 X R_{A B M N} R^{A B M N}\right),
\end{aligned}
$$

where $I$ is the $N \times N$ identity matrix.

\section{References}

[1] S. Weinberg, Rev. Mod. Phys. 61 (1989) 1.

[2] S. Perlmutter et al., Ap. J. 483565 (1997) [astro-ph/9712212];

A.G. Riess et al, Ast. J. 1161009 (1997) [astro-ph/9805201];

N. Bahcall, J.P. Ostriker, S. Perlmutter, P.J. Steinhardt, Science 284 (1999) 1481, [astro-ph/9906463].

[3] G. 't Hooft, in Cargese Summer Inst. 1979:135 (QCD161:S77:1979) (reprinted in 't Hooft, G. (ed.): Under the spell of the gauge principle 352-374, and in Farhi, E. (ed.), Jackiw, R. (ed.): Dynamical gauge symmetry breaking 345-367).

\footnotetext{
${ }^{12}$ In comparing with this reference recall that our metric is 'mostly plus' and we adopt Weinberg's curvature conventions [17, which for the Riemann tensor agree with those of ref. [16], but disagree with this reference by a sign for the Ricci tensor and scalar.
} 
[4] For a review with references see J. Polchinski, TASI Lectures on D-Branes [hep-th/9611050].

[5] N. Arkani-Hamed, S. Dimopoulos and G. Dvali, Phys. Lett. B429 (1998) 263 [hep-ph/9803315]; Phys. Rev. D59 (1999) 086004 [hep-ph/9807344].

[6] Y. Aghababaie, C.P. Burgess, S. Parameswaran and F. Quevedo, Nucl. Phys. B680 (2004) 389-414, [hep-th/0304256].

[7] Y. Aghabababie, C.P. Burgess, J.M. Cline, H. Firouzjahi, S. Parameswaran, F. Quevedo, G. Tasinato and I. Zavala, JHEP 0309 (2003) 037 (48 pages)

[hep-th/0308064];

G. Azuelos, P.H. Beauchemin and C.P. Burgess, [hep-ph/0401125];

C.P. Burgess, J. Matias and F. Quevedo, [hep-ph/0404135];

P.H. Beauchemin, G. Azuelos and C.P. Burgess, J. Phys. G30 (2004) N17

[hep-ph/0407196];

C.P. Burgess, F. Quevedo, G. Tasinato and I. Zavala, [hep-th/0408109].

[8] For reviews of the SLED proposal see: C.P. Burgess, Ann. Phys. 313 (2004) 283-401 [hep-th/0402200]; and in the proceedings of the Texas A\&M Workshop on String Cosmology, [hep-th/0411140].

[9] S. M. Carroll and M. M. Guica, [hep-th/0302067];

I. Navarro, JCAP 0309 (2003) 004 [hep-th/0302129];

I. Navarro, Class. Quant. Grav. 20 (2003) 3603 [hep-th/0305014];

G. W. Gibbons, R. Guven and C. N. Pope, Phys. Lett. B 595 (2004) 498 [hep-th/0307238];

H. P. Nilles, A. Papazoglou and G. Tasinato, Nucl. Phys. B 677 (2004) 405 [hep-th/0309042];

I. Navarro and J. Santiago, JHEP 0404, 062 (2004) [arXiv:hep-th/0402204];

A. L. Maroto, Phys. Rev. D 69, 101304 (2004) [arXiv:hep-ph/0402278];

S. Randjbar-Daemi and E. Sezgin, Nucl. Phys. B 692, 346 (2004) [arXiv:hep-th/0402217];

M. L. Graesser, J. E. Kile and P. Wang, Phys. Rev. D 70, 024008 (2004) [arXiv:hep-th/0403074];

C. Kim, Y. Kim and O. K. Kwon, JHEP 0405, 020 (2004) [arXiv:hep-th/0404163];

I. Navarro and J. Santiago, JCAP 0409 (2004) 005 [hep-th/0405173];

A. Kehagias, Phys. Lett. B 600, 133 (2004) [arXiv:hep-th/0406025];

J. Vinet and J. M. Cline, [hep-th/0406141];

J. Garriga and M. Porrati, [hep-th/0406158];

T. Biswas and P. Jaikumar, JHEP 0408, 053 (2004) [arXiv:hep-th/0407063];

S. Randjbar-Daemi and V. Rubakov, [hep-th/0407176];

H. M. Lee and A. Papazoglou, [hep-th/0407208]; 
V.P. Nair and S. Randjbar-Daemi, [hep-th/0408063];

R. Erdem, [arXiv:hep-th/0410063];

S. Nobbenhuis, [arXiv:gr-qc/0411093];

P. Brax, C. van de Bruck and A. C. Davis, [arXiv:hep-th/0411208];

I. Navarro and J. Santiago, [hep-th/0411250];

K. Kainulainen and D. Sunhede, arXiv:astro-ph/0412609;

M. Redi, Phys. Rev. D 71, 044006 (2005) [arXiv:hep-th/0412189];

G. Kofinas, [arXiv:hep-th/0412299];

J. M. Schwindt and C. Wetterich, [arXiv:hep-th/0501049];

E. Papantonopoulos and A. Papazoglou, [arXiv:hep-th/0501112];

C. Charmousis and R. Zegers, [arXiv:hep-th/0502170].

[10] J. Vinet and J. Cline, [hep-th/0501098].

[11] P. Candelas and S. Weinberg, "Calculation Of Gauge Couplings And Compact Circumferences From Selfconsistent Dimensional Reduction," Nucl. Phys. B 237 (1984) 397;

C. R. Ordonez and M. A. Rubin, Nucl. Phys. B 260 (1985) 456;

UTTG-18-84-ERRATUM;

R. Kantowski and K.A. Milton, Phys. Rev. D35 (1987) 549;

D. Birmingham, R. Kantowski and K.A. Milton, Phys. Rev. D38 (1988) 1809;

C. C. Lee and C. L. Ho, "Symmetry breaking by Wilson lines and finite temperature and density effects," Mod. Phys. Lett. A 8, 1495 (1993);

M. Ito, "Casimir forces due to matters in compactified six dimensions," Nucl. Phys. B 668 (2003) 322 [hep-ph/0301168];

E. Ponton and E. Poppitz, "Casimir energy and radius stabilization in five and six dimensional orbifolds," JHEP 0106 (2001) 019 [hep-ph/0105021];

S. Matsuda and S. Seki, "Cosmological constant probing shape moduli through large extra dimensions," hep-th/0404121;

Y. Cho and K. Shiraishi, [arXiv:hep-th/0405154];

Y. Hosotani, S. Noda and K. Takenaga, "Dynamical gauge symmetry breaking and mass generation on the orbifold $T^{* * 2 / Z(2) ", ~ h e p-p h / 0403106 ; ~}$

Y. Hosotani, S. Noda and K. Takenaga, "Dynamical gauge-Higgs unification in the electroweak theory," hep-ph/0410193;

I. Antoniadis, K. Benakli and M. Quiros, "Finite Higgs mass without supersymmetry," New J. Phys. 3 (2001) 20 [hep-th/0108005];

J. E. Hetrick and C. L. Ho, "Dynamical Symmetry Breaking From Toroidal Compactification," Phys. Rev. D 40, 4085 (1989);

C. C. Lee and C. L. Ho, "Recurrent dynamical symmetry breaking and restoration by Wilson lines at finite densities on a torus," Phys. Rev. D 62, 085021 (2000)

[hep-th/0010162]; 
A. Albrecht, C. P. Burgess, F. Ravndal and C. Skordis, "Exponentially large extra dimensions," Phys. Rev. D 65 (2002) 123506 [hep-th/0105261];

E. Elizalde, K. Kirsten and Y. Kubyshin, "On the instability of the vacuum in multidimensional scalar theories," Z. Phys. C 70 (1996) 159 [hep-th/9410101];

N. Haba, M. Harada, Y. Hosotani and Y. Kawamura, "Dynamical rearrangement of gauge symmetry on the orbifold $S^{* * 1 / Z(2), " ~[h e p-p h / 0212035] ; ~}$

D.M. Ghilencea, C.P. Burgess and F. Quevedo, "Casimir Energies for 6D Supergravities Compactified on 2-Tori and Their Orbifolds," (to appear).

[12] D. Hoover et al., "Casimir Energy and UV Sensitivity in 6D Supergravity with Fluxes," (in preparation).

[13] P. B. Gilkey, J. Diff. Geom. 10, 601 (1975).

[14] S.M. Christensen, Phys. Rev. D17 (1978) 9460-963; S.M. Christensen and M.J. Duff, Nucl. Phys. B154 (1979) 301; D.M. McAvity and H. Osborn, Class. Quant. Grav. 8 (1991) 603-638.

[15] M.J. Duff and P. van Nieuwenhuizen, "Quantum Inequivalence of Different Field Representations," Phys. Lett. B94 (1980) 179;

S. M. Christensen, M. J. Duff, G. W. Gibbons and M. Rocek, "Vanishing One Loop Beta Function In Gauged N>4 Supergravity," Phys. Rev. Lett. 45 (1980) 161;

M. J. Duff, Presented at Nuffield Supergravity Workshop, Cambridge, England, Jun 22 - Jul 12, 1980.

[16] For a recent review of heat kernel techniques see: D. V. Vassilevich, "Heat kernel expansion: User's manual," Phys. Rept. 388, 279 (2003) [arXiv:hep-th/0306138].

[17] S. Weinberg, Gravitation and Cosmology, Wiley, New York, 1972.

[18] C.W. Misner, J.A. Wheeler and K.S. Thorne, Gravitation, W.H. Freeman and Co., 1973.

[19] G.H. Hardy, Divergent Series, Oxford University Press, 1949.

[20] S.W. Hawking, "Zeta Function Regularization of Path Integrals in Curved Space-Time,", Commun. Math. Phys. 55 (1977) 133.

[21] N. D. Birrell and P. C. W. Davies, Quantum Fields In Curved Space, Cambridge University Press, 1982.

[22] I.S. Gradshteyn, I.M.Ryzhik, "Table of Integrals, Series and Products", Academic Press Inc., New York/London, 1965.

[23] A. Vilenkin, Phys. Rev. D23 (1981) 852;

R. Gregory and C. Santos, Phys. Rev. D56, 1194 (1997) [gr-qc/9701014]. 
[24] C.P. Burgess, F. Quevedo, G. Tasinato and I. Zavala, JHEP (2004) 0411:069 [hep-th/0408109].

[25] J.S. Dowker, Phys. Rev. D36 (1987) 3095;

D. Kabat, Nucl. Phys. B 453, 281 (1995) [hep-th/9503016].

[26] D. V. Fursaev and S. N. Solodukhin, Phys. Rev. D 52, 2133 (1995) [hep-th/9501127];

L. De Nardo, D. V. Fursaev and G. Miele, Class. Quant. Grav. 14, 1059 (1997) [hep-th/9610011].

[27] D. V. Fursaev and G. Miele, Nucl. Phys. B 484, 697 (1997) [hep-th/9605153].

[28] Irrelevant interactions commonly arise within effective fields theories, and are described in: C.P. Burgess, Goldstone and Pseudo-Goldstone Bosons in Nuclear, Particle and Condensed-Matter Physics, Physics Reports 330 (2000) 193-261, (hep-ph/9808176); in *Barcelona 1998, Radiative corrections: Application of quantum field theory to phenomenology* 471-488 [hep-ph/9812470];

Living Rev. Rel. 7 (2004) 5 [gr-qc/0311082].

[29] Some textbooks which describe Effective lagrangians are:

Weak Interactions and Modern Particle Theory, Howard Georgi,

Benjamin/Cummings, 1984; Dynamics of the Standard Model, John F. Donoghue, Eugene Golowich and Barry R. Holstein, Cambridge University Press, 1992.

S. Weinberg, ref. [31].

[30] E. S. Abers and B. W. Lee, Phys. Rept. 9, 1 (1973).

[31] S. Weinberg, The quantum theory of fields, Vol. I: Foundations, Cambridge University Press (1995); The quantum theory of fields, Vol II: Modern Applications, Cambridge University Press (1996).

[32] D. Hoover et. al., "Heat Kernel Coefficients for General Spins in Higher Dimensions," (in preparation).

[33] J. Strathdee, Int. J. Mod. Phys. A 2, 273 (1987).

[34] J. Scherk and J.H. Schwarz, Phys. Lett. B82 (1979) 60.

[35] S. Randjbar-Daemi, A. Salam and J. Strathdee, Nucl. Phys. B 214 (1983) 491. S. Randjbar-Daemi, A. Salam, E. Sezgin and J. Strathdee, Phys. Lett. B151 (1985) 351.

[36] N. Marcus and J.H. Schwarz, Phys. Lett. 115B (1982) 111; H. Nishino and E. Sezgin, Phys. Lett. 144B (1984) 187; "The Complete N=2, D = 6 Supergravity With Matter And Yang-Mills Couplings," Nucl. Phys. B278 (1986) 353; Nucl. Phys. B 278 (1986) 353; Nucl. Phys. B 505 (1997) 497 [hep-th/9703075]. 
[37] P. Candelas and S. Weinberg, Nucl. Phys. B237 (1984) 397;

R. Kantowski and K.A. Milton, Phys. Rev. D35 (1987) 549;

D. Birmingham, R. Kantowski and K.A. Milton, Phys. Rev. D38 (1988) 1809.

[38] M.B. Green, J.H. Schwarz and P.C. West, Nucl. Phys. B254 (1985) 327;

J. Erler, J. Math. Phys. 35 (1994) 1819 [hep-th/9304104].

[39] Y. Aghababaie, C.P. Burgess, S. Parameswaran and F. Quevedo, "Supersymmetry Breaking and Moduli Stabilization from Fluxes and Six-Dimensional Supergravity," JHEP 0303 (2003) 032, [hep-th/0212091].

[40] E. S. Fradkin and A. A. Tseytlin, Phys. Lett. B 123, 231 (1983).

[41] S. Weinberg, Phys. Lett. B91 (1980) 51; B.A. Ovrut and H.J. Schnitzer, Phys. Lett. B100 (1981) 403-406; Nucl. Phys. B179 (1981) 381-416.

[42] C.P. Burgess and D. London, "On Anomalous Gauge Boson Couplings and Loop Calculations," Phys. Rev. Lett. 69 (1992) 3428-3431; "Uses and Abuses of Effective Lagrangians," Phys. Rev. D48 (1993) 4337- 4351. 